\title{
Abelian coverings of finite general linear groups and an application to their non-commuting graphs
}

\author{
Azizollah Azad • Mohammad A. Iranmanesh • \\ Cheryl E. Praeger · Pablo Spiga
}

Received: 24 October 2010 / Accepted: 5 April 2011 / Published online: 29 April 2011

(C) Springer Science+Business Media, LLC 2011

\begin{abstract}
In this paper we introduce and study a family $\mathcal{A}_{n}(q)$ of abelian subgroups of $\mathrm{GL}_{n}(q)$ covering every element of $\mathrm{GL}_{n}(q)$. We show that $\mathcal{A}_{n}(q)$ contains all the centralizers of cyclic matrices and equality holds if $q>n$. For $q>2$, we obtain an infinite product expression for a probabilistic generating function for $\left|\mathcal{A}_{n}(q)\right|$. This leads to upper and lower bounds which show in particular that
\end{abstract}

$$
c_{1} q^{-n} \leq \frac{\left|\mathcal{A}_{n}(q)\right|}{\left|\mathrm{GL}_{n}(q)\right|} \leq c_{2} q^{-n}
$$

for explicit positive constants $c_{1}, c_{2}$. We also prove that similar upper and lower bounds hold for $q=2$.

For the 100th anniversary of the birth of B.H. Neumann.

The paper forms part of the Australian Research Council Federation Fellowship Project FF0776186 of the third author. The fourth author is supported by UWA as part of the Federation Fellowship project. The second author is supported by Research Council of Yazd University.

A. Azad $(\bowtie)$

Department of Mathematics, Faculty of Sciences, Arak University, Arak 38156-8-8349, Iran

e-mail: a-azad@araku.ac.ir

M.A. Iranmanesh

Department of Mathematics, Yazd University, Yazd 89195-741, Iran

e-mail: iranmanesh@yazduni.ac.ir

C.E. Praeger · P. Spiga

School of Mathematics and Statistics, The University of Western Australia, Crawley, WA 6009, Australia

C.E. Praeger

e-mail: praeger@maths.uwa.edu.au

P. Spiga

e-mail: spiga@maths.uwa.edu.au 
A subset $X$ of a finite group $G$ is said to be pairwise non-commuting if $x y \neq y x$ for distinct elements $x, y$ in $X$. As an application of our results on $\mathcal{A}_{n}(q)$, we prove lower and upper bounds for the maximum size of a pairwise non-commuting subset of $\mathrm{GL}_{n}(q)$. (This is the clique number of the non-commuting graph.) Moreover, in the case where $q>n$, we give an explicit formula for the maximum size of a pairwise non-commuting set.

Keywords General linear group - Cyclic matrix - Non-commuting subsets of finite groups $\cdot$ Non-commuting graph

\section{Introduction}

In a finite general linear group $\mathrm{GL}_{n}(q)$ the class of cyclic matrices (see Sect. 1.1 for the definition) plays an important role both algorithmically (see [15]) and in representation theory (for the recognition of irreducible representations). First we use explicit lower bounds for the proportion of cyclic matrices in $\mathrm{GL}_{n}(q)$ (obtained in $[9,14$, 20]) to determine a lower bound for the maximum size $\omega\left(\mathrm{GL}_{n}(q)\right)$ of a set of pairwise non-commuting elements of $\mathrm{GL}_{n}(q)$, or equivalently the clique number of the non-commuting graph for this group. The study of these clique numbers for various families of groups goes back to the 1976 paper [13] of B.H. Neumann, answering a question of Paul Erdös, and inspired much subsequent research. A short account is given in Sect. 1.1 to contextualise our results. Our paper is written in honour of the 100th anniversary of B.H. Neumann's birth on 15 October 1909.

Theorem 1.1 For $q \geq 2$,

$$
q^{-n}\left(1-q^{-3}-q^{-5}+q^{-6}-q^{-n}\right)<\frac{\omega\left(\mathrm{GL}_{n}(q)\right)}{\left|\mathrm{GL}_{n}(q)\right|} \leq q^{-n} l(q)
$$

where

$$
l(q)=\prod_{k=1}^{\infty}\left(1-q^{-k}\right)^{-k(k+1) / 2-1} \leq \begin{cases}1+2 q^{-1}+7 q^{-2}+114 q^{-3} & \text { if } q \geq 3 \\ 395.0005 & \text { if } q=2\end{cases}
$$

We comment separately on our strategies for proving the lower bound (in Sect. 1.1) and the upper bound (in Sect. 1.2). In particular, deriving the upper bound involves a natural family of abelian subgroups of $\mathrm{GL}_{n}(q)$ covering every group element. Detailed upper and lower bounds for the infinite product $l(q)$ are obtained in Lemma 7.1.

\subsection{Cyclic matrices and the lower bound}

An element $g \in \mathrm{GL}_{n}(q)$ is cyclic if its characteristic polynomial is equal to its minimal polynomial.

Definition 1.2 We denote by $\mathcal{N}_{n}(q)$ the set of centralisers of cyclic matrices in $\mathrm{GL}_{n}(q)$, that is, $\mathcal{N}_{n}(q)=\left\{C_{\mathrm{GL}_{n}(q)}(g) \mid g\right.$ cyclic in $\left.\mathrm{GL}_{n}(q)\right\}$. Also, we denote by $N_{n}(q)$ the cardinality $\left|\mathcal{N}_{n}(q)\right|$. 
The centralisers of cyclic matrices in $\mathrm{GL}_{n}(q)$ are abelian and small (when compared with the centralisers of non-cyclic matrices). Moreover, they cover a large fraction of the elements of $\mathrm{GL}_{n}(q)$. It turns out that results of Neumann and Praeger [14], and more precise results obtained independently by Fulman [9] and Wall [20], can be applied almost directly to give very good lower bounds on $N_{n}(q)$ and on $\omega\left(\operatorname{GL}_{n}(q)\right)$.

\section{Theorem 1.3}

$$
\frac{\omega\left(\mathrm{GL}_{n}(q)\right)}{\left|\mathrm{GL}_{n}(q)\right|} \geq \frac{N_{n}(q)}{\left|\mathrm{GL}_{n}(q)\right|}>q^{-n}\left(1-q^{-3}-q^{-5}+q^{-6}-q^{-n}\right) .
$$

\subsection{An Abelian covering and the upper bound}

For some $n, q$, the centralisers of cyclic matrices do not cover every element in $\mathrm{GL}_{n}(q)$ (see Remark 3.3 at the end of Sect. 3). Therefore, in Definition 1.5 below we define a family $\mathcal{A}_{n}(q)$ of abelian subgroups of $\mathrm{GL}_{n}(q)$, which contains $\mathcal{N}_{n}(q)$ and covers every element of $\mathrm{GL}_{n}(q)$, giving an upper bound for $\omega\left(\mathrm{GL}_{n}(q)\right)$. We prove moreover that $\mathcal{N}_{n}(q)=\mathcal{A}_{n}(q)$ for $q>n$.

\section{Theorem 1.4}

(a) $\operatorname{GL}_{n}(q)=\bigcup_{A \in \mathcal{A}_{n}(q)} A$.

(b) $\omega\left(\mathrm{GL}_{n}(q)\right) \leq\left|\mathcal{A}_{n}(q)\right|$ with equality if and only if $q>n$.

(c) $\mathcal{N}_{n}(q) \subseteq \mathcal{A}_{n}(q)$ with equality if and only if $q>n$.

Thus an expression for $\left|\mathcal{A}_{n}(q)\right|$, or even a good upper bound, provides an upper bound for $\omega\left(\mathrm{GL}_{n}(q)\right)$. It seems, on consulting several of our colleagues, that the family $\mathcal{A}_{n}(q)$ has not been studied previously. This surprised us, considering the central role it plays in Theorem 1.4. The set $\mathcal{A}_{n}(q)$ is defined as follows.

Definition 1.5 Let $V$ be the $n$-dimensional vector space $k^{n}$ over the field $k$ of size $q$. Let $\mathcal{A}_{n}(q)$ be the set of abelian subgroups $A$ of $\mathrm{GL}_{n}(q)$ such that the $A$-module $V$ has a decomposition $V_{1} \oplus \cdots \oplus V_{r}$ into indecomposable $A$-modules satisfying the following properties:

(i) $A=A_{1} \times \cdots \times A_{r}$, where $A_{i} \subseteq \mathrm{GL}\left(V_{i}\right)$.

(ii) for $i=1, \ldots, r$, we have $A_{i}=C_{\mathrm{GL}\left(V_{i}\right)}\left(a_{i}\right)$ for some element $a_{i} \in \mathrm{GL}\left(V_{i}\right)$ such that $V_{i}$ is an indecomposable $\left\langle a_{i}\right\rangle$-module.

It is shown in Proposition 5.7 that, for $q>2$, the elements in $\mathcal{A}_{n}(q)$ are maximal abelian subgroups of $\mathrm{GL}_{n}(q)$. The bulk of the paper is devoted to determining the limiting size of $\mathcal{A}_{n}(q)$ for a fixed field size $q>2$ and large $n$.

Theorem 1.6 For $q>2$, the sequence $\left\{q^{n}\left|\mathcal{A}_{n}(q)\right| /\left|\mathrm{GL}_{n}(q)\right|\right\}_{n \geq 0}$ is increasing with limit $l(q)$, as defined in (1.1), and we have

$$
\left|q^{n} \frac{\left|\mathcal{A}_{n}(q)\right|}{\left|\mathrm{GL}_{n}(q)\right|}-l(q)\right|=o\left(r^{-n / 2}\right)
$$


for any $r$ such that $1<r<q$. For $q=2$, there exists an increasing sequence $\left\{2^{n} b_{n}\right\}_{n \geq 0}$ with limit $l(2)$ such that $\left|\mathcal{A}_{n}(2)\right| /\left|\mathrm{GL}_{n}(2)\right| \leq b_{n}$, and we have

$$
\left|2^{n} b_{n}-l(2)\right|=o\left(r^{-n / 2}\right)
$$

for any $r$ such that $1<r<2$.

The sequence $\left\{b_{n}\right\}_{n \geq 1}$ can be found in Definition 6.1. Our method is to study the generating function $F(t)=\sum_{n}^{\infty} a_{n} t^{n}$, where $a_{n}=\left|\mathcal{A}_{n}(q)\right| /\left|\mathrm{GL}_{n}(q)\right|$ and $a_{0}=1$ (see Definition 6.1). For $q>2$, we obtain a simple formula for $F(t)$. In fact, using the theory of symmetric functions, we prove the following result.

Theorem 1.7 If $q>2$, then

$$
F(t)=\left(\prod_{i=0}^{\infty}\left(1-q^{-(i+1)} t\right)^{-1}\right)\left(\prod_{m \geq 2, i, j \geq 0}\left(1-q^{-(i+j+2 m-1)} t^{m}\right)^{-1}\right) .
$$

Moreover, $F(t)$ has a simple pole at $t=q$, and $\left(1-q^{-1} t\right) F(t)$ is analytic on a disk of radius $q^{3 / 2}$.

It would be interesting to know if a similar formula could be obtained for $F(t)$ when $q=2$.

Remark 1.8 It follows from Theorems 1.1, 1.3, and 1.4(b) that

$$
c_{1} q^{-n} \leq\left|\mathcal{A}_{n}(q)\right| /\left|\mathrm{GL}_{n}(q)\right| \leq c_{2} q^{-n}
$$

for explicit positive constants $c_{1}, c_{2}$.

The coefficient of degree $n$ in $F(t)$ equals $\left|\mathcal{A}_{n}(q)\right| /\left|\mathrm{GL}_{n}(q)\right|$, which also equals $\omega\left(\mathrm{GL}_{n}(q)\right) /\left|\mathrm{GL}_{n}(q)\right|$ when $q>n$, by Theorem 1.4(b). In particular, the equation for $F(t)$ in Theorem 1.7 can be used to obtain explicit formulas for $\omega\left(\mathrm{GL}_{n}(q)\right)$ when $q>n$.

We present in Table 1 the values of $\left|\mathcal{A}_{n}(q)\right|$ for $1 \leq n \leq 6$ and $q>2$.

In the next subsection, we recall how the problem of determining the maximum size $\omega(G)$ of a pairwise non-commuting set of elements arises in group theory. Also, we recall some results on $\omega(G)$ for various families of groups, and we see how our method generalises some results in the literature $[1,2]$.

\subsection{The non-commuting graph of a group}

In 1976, Neumann [13] answered a question of Paul Erdős about the maximal clique size in the non-commuting graph $\Gamma(G)$ of a group $G$, namely the graph with vertices the elements of $G$ and with edges the pairs $\{x, y\}$ with $x y \neq y x$. A clique in a graph is a set of pairwise adjacent vertices, and hence in $\Gamma(G)$ a clique is a pairwise noncommuting subset of $G$. In group theoretic language, Erdős asked whether there exists a finite upper bound on the cardinalities of pairwise non-commuting subsets of $G$, 


\section{Table 1}

\begin{tabular}{|c|c|}
\hline$n$ & $\left|\mathcal{A}_{n}(q)\right|$ \\
\hline 1 & 1 \\
\hline 2 & $q^{2}+q+1$ \\
\hline 3 & $q^{6}+q^{5}+3 q^{4}+3 q^{3}+q^{2}-q-1$ \\
\hline 4 & $q^{12}+q^{11}+4 q^{10}+7 q^{9}+9 q^{8}+5 q^{7}+2 q^{6}-3 q^{5}-2 q^{4}-q^{3}+q^{2}+q$ \\
\hline 5 & $\begin{array}{l}q^{20}+q^{19}+4 q^{18}+9 q^{17}+18 q^{16}+22 q^{15}+22 q^{14}+15 q^{13}+6 q^{12}-4 q^{11}-7 q^{10} \\
\quad-6 q^{9}-2 q^{8}+q^{7}+2 q^{6}+q^{5}-q^{4}-q^{3}\end{array}$ \\
\hline 6 & $\begin{array}{l}q^{30}+q^{29}+4 q^{28}+10 q^{27}+23 q^{26}+40 q^{25}+60 q^{24}+65 q^{23}+68 q^{22}+53 q^{21} \\
\quad+33 q^{20}+5 q^{19}-8 q^{18}-19 q^{17}-16 q^{16}-7 q^{15}+q^{14}+6 q^{13}+6 q^{12}+5 q^{11} \\
\quad-q^{9}-q^{8}+q^{7}+q^{6}\end{array}$ \\
\hline
\end{tabular}

Table 2 Results for $\omega(\operatorname{Sym}(n))$ from Brown [4]

\begin{tabular}{ll}
\hline Lower bound & Comments \\
\hline$a(n-2) !$ & $\begin{array}{l}\text { for every } n \\
\text { for infinitely many } n\end{array}$ \\
$d(\log \log n)(n-2) !$ & \\
\hline Upper bound & for every $n$ \\
\hline$b(\log \log n)(n-2) !$ & for infinitely many $n$ \\
$c(n-2) !$ &
\end{tabular}

assuming that every such subset is finite. Neumann proved that the family of groups satisfying the condition of Erdős is precisely the class of groups $G$ in which the centre $Z(G)$ has finite index, and he proved moreover that for such groups $G$, each pairwise non-commuting subset of $G$ has size at most $|G: Z(G)|-1$. Neumann's answer inspired much subsequent research, for example [1-4, 6, 12, 16, 18].

We let $\omega(G)$ denote the maximum cardinality of a pairwise non-commuting subset of $G$. Because of Neumann's result, the study of groups $G$ such that $\omega(G)<\infty$ is reduced to the study of finite groups. It follows from results of [16] that, if $n=\mid G$ : $Z(G) \mid$, then $c \log _{2} n \leq \omega(G) \leq n-1$ for some positive constant $c$. The lower bound is achieved with $c=1$ by each extraspecial 2-group. (According to [3, 16], this was proved by Isaacs.)

On the other hand, the upper bound is achieved for the quaternion group $G=Q_{8}$ and the dihedral group $G=D_{8}$ of order 8 , both of which have $\omega(G)=3$. It is believed that groups $G$ which are "close" to being nonabelian simple will have $\omega(G)$ "close" to the upper bound. Indeed, for the symmetric group $\operatorname{Sym}(n)$ of degree $n, \omega(\operatorname{Sym}(n))$ satisfies the bounds in Table 2, where $a, b, c, d$ are constants, see [4, Theorem 1].

Also, for finite general linear groups $\mathrm{GL}_{n}(q)$, if $q>2$, then $\omega\left(\mathrm{GL}_{2}(q)\right)=q^{2}+$ $q+1$ (see [1, Lemma 4.4]), and if $q>3$, then $\omega\left(\operatorname{GL}_{3}(q)\right)=q^{6}+q^{5}+3 q^{4}+3 q^{3}+$ $q^{2}-q-1$ (see [2, Theorem 1.1]). These two results on the finite general linear group prove the bounds of Theorem 1.1 for $n \leq 3$, and the values for $\omega\left(\mathrm{GL}_{n}(q)\right.$ ) (for $n=2,3$ ) are exactly the second and third rows in Table 1. 
Finally, it was conjectured [2, Conjecture 1.2] that, for $q>n$, the number $\omega\left(\mathrm{GL}_{n}(q)\right)$ should be somewhat larger than $q^{n^{2}-n}+q^{n^{2}-n-1}+(n-1) q^{n^{2}-n-2}$. As we discuss in Remark 7.6, this conjecture is incorrect for $n \geq 6$.

Using the results of [10], a lower bound for $\omega(G)$ similar to that provided by Theorem 1.1 can be obtained for all finite classical groups $G$. It would be interesting to know if a similar estimation for a class of abelian subgroups of classical groups could be carried out to yield a good upper bound for $\omega(G)$ for these groups.

\subsection{Structure of the paper}

In this final introductory section we give brief summary proofs of the major results and in so doing indicate where the relevant technical results can be found.

Proof of Theorem 1.1 The lower bound is a direct application of Theorem 1.3. From Theorem 1.4 we have $\omega\left(\mathrm{GL}_{n}(q)\right) \leq\left|\mathcal{A}_{n}(q)\right|$, and from Theorem 1.6 the sequence $\left\{q^{n}\left|\mathcal{A}_{n}\right| /\left|\mathrm{GL}_{n}(q)\right|\right\}_{n}$ is increasing with limit $l(q)$. Therefore the upper bound follows. The estimates on $l(q)$ are collected in Lemma 7.1 in Sect. 7.

Proof of Theorem 1.3 The proof of this result is given in Sect. 2.

Proof of Theorem 1.4 This theorem is proved in Sect. 5. Namely, Part (a) is proved in Proposition 5.1, Part (b) (which is an application of Part (a)) is proved in Corollary 5.10, and Part (c) is proved in Theorem 5.9.

Proof of Theorem 1.6 This result is proved in Sects. 6 and 7. Namely, in Theorem 6.7 we prove that the sequences $\left\{q^{n}\left|\mathcal{A}_{n}(q)\right| /\left|\mathrm{GL}_{n}(q)\right|\right\}_{n}$ (for $q>2$ ) and $\left\{q^{n} b_{n}\right\}_{n}$ (for $q=2$ ) are increasing. In Theorem 7.4 we compute the rate of convergence and the limit.

Proof of Theorem 1.7 The equation for the generating function $F(t)$ is proved in Theorem 6.6. The rest of the theorem follows from Propositions 7.2 and 7.3.

\section{Lower bound: proof of Theorem 1.3}

Recall that an element $g$ in $\mathrm{GL}_{n}(q)$ is said to be a cyclic matrix if the characteristic polynomial of $g$ is equal to its minimum polynomial, see [14]. If $g$ is a cyclic matrix, then (see [14, Theorem 2.1(3)]) the group $C_{\mathrm{GL}_{n}(q)}(g)$ is abelian, and by [14, Corollary 2.3] we have $\left|C_{\mathrm{GL}_{n}(q)}(g)\right| \leq q^{n}$. Thus the groups in $\mathcal{N}_{n}(q)$ (see Definition 1.2) are abelian of order at most $q^{n}$.

Cyclic matrices of $\mathrm{GL}_{n}(q)$ are well studied (see [10, 14]), and in particular Wall (see [10, page 2]) proved that the proportion $c_{\mathrm{GL}}(n, q)$ of cyclic matrices in $\operatorname{GL}_{n}(q)$ satisfies

$$
\left|c_{\mathrm{GL}}(n, q)-\frac{1-q^{-5}}{1+q^{-3}}\right| \leq \frac{1}{q^{n}(q-1)} .
$$


Thus,

$$
c_{\mathrm{GL}}(n, q) \geq \frac{1-q^{-5}}{1+q^{-3}}-\frac{1}{q^{n}(q-1)}>1-q^{-3}-q^{-5}+q^{-6}-q^{-n},
$$

where the second inequality is obtained by expanding $\left(1-q^{-5}\right) /\left(1+q^{-3}\right)$ in powers of $q$ and by noticing that $1 / q^{n}(q-1) \leq 1 / q^{n}$. Using this remarkable result, we easily obtain Theorem 1.3.

Proof of Theorem 1.3 Let $\mathcal{C}_{n}(q)$ denote the set of cyclic matrices of $\mathrm{GL}_{n}(q)$ and $X=\left\{(g, C) \mid g \in \mathcal{C}_{n}(q), C \in \mathcal{N}_{n}(q), g \in C\right\}$. We claim that every element $g$ of $\mathcal{C}_{n}(q)$ lies in a unique element of $\mathcal{N}_{n}(q)$. Indeed, assume that $g \in C_{1}, C_{2}$ for some $C_{1}, C_{2} \in \mathcal{N}_{n}(q)$, and let $g_{1}, g_{2}$ be cyclic matrices such that $C_{i}=C_{\mathrm{GL}_{n}(q)}\left(g_{i}\right)$ for $i=1,2$. As $C_{1}, C_{2}$ are abelian and $g \in C_{1}, C_{2}$, we get $C_{1}, C_{2} \subseteq C_{\mathrm{GL}_{n}(q)}(g)$. Similarly, as $C_{\mathrm{GL}_{n}(q)}(g)$ is abelian and $g_{i} \in C_{i} \subseteq C_{\mathrm{GL}_{n}(q)}(g)$, we get $C_{\mathrm{GL}_{n}(q)}(g) \subseteq C_{i}$ and $C_{\mathrm{GL}_{n}(q)}(g)=C_{1}=C_{2}$.

Counting the size of the set $X$, we have

$$
\begin{aligned}
q^{n} N_{n}(q) & =q^{n}\left|\mathcal{N}_{n}(q)\right|=\sum_{C \in \mathcal{N}_{n}(q)} q^{n} \geq \sum_{C \in \mathcal{N}_{n}(q)}\left|C \cap \mathcal{C}_{n}(q)\right|=|X| \\
& =\sum_{g \in \mathcal{C}_{n}(q)}\left|\left\{C \in \mathcal{N}_{n}(q) \mid g \in C\right\}\right| \\
& =\sum_{g \in \mathcal{C}_{n}(q)} 1=\left|\mathcal{C}_{n}(q)\right|=\left|\mathrm{GL}_{n}(q)\right| c_{\mathrm{GL}}(n, q) .
\end{aligned}
$$

Now (2.1) yields $N_{n}(q)>q^{-n}\left|\mathrm{GL}_{n}(q)\right|\left(1-q^{-3}-q^{-5}+q^{-6}-q^{-n}\right)$.

It remains to prove that $\omega\left(\mathrm{GL}_{n}(q)\right) \geq N_{n}(q)$. Let $\mathcal{N}_{n}(q)=\left\{C_{1}, \ldots, C_{r}\right\}$ with $r=N_{n}(q)$. Let $g_{i}$ be a cyclic matrix in $\operatorname{GL}_{n}(q)$ such that $C_{i}=C_{\mathrm{GL}_{n}(q)}\left(g_{i}\right)$ for $i=1, \ldots, r$. Set $S=\left\{g_{i} \mid 1 \leq i \leq r\right\}$. We claim that, if $i \neq j$, then the group elements $g_{i}$ and $g_{j}$ of $S$ do not commute. If $g_{i} g_{j}=g_{j} g_{i}$, then $g_{j} \in C_{\mathrm{GL}_{n}(q)}\left(g_{i}\right)=C_{i}$, whereas we showed above that $C_{j}$ is the unique element of $\mathcal{N}_{n}(q)$ containing $g_{j}$. This yields $\omega\left(\mathrm{GL}_{n}(q)\right) \geq|S|=r=N_{n}(q)$, and thus the theorem follows.

\section{Upper bound: idea of the proof}

In the rest of this paper, we determine an upper bound for $\omega\left(\mathrm{GL}_{n}(q)\right.$ ) (and hence for $N_{n}(q)$ by Theorem 1.3). Also, for $q>n$, we prove that $N_{n}(q)=\omega\left(\mathrm{GL}_{n}(q)\right)$, and we obtain an explicit formula for $N_{n}(q)$. Before going into more detail, in this section we briefly describe the method that is used. First, our results rely on this elementary observation.

Lemma 3.1 Let $G$ be a group, and $\mathcal{A}$ be a collection of abelian subgroups of $G$ such that $G=\bigcup_{A \in \mathcal{A}}$ A. Then $\omega(G) \leq|\mathcal{A}|$. 
Proof Let $S$ be a pairwise non-commuting set. Since $A \in \mathcal{A}$ is abelian, we get $\mid S \cap$ $A \mid \leq 1$. As $G=\bigcup_{A \in \mathcal{A}} A$, we obtain $|S| \leq|\mathcal{A}|$. Thus the result follows.

Lemma 3.1 can be used effectively to obtain upper bounds for $\omega(G)$. As an example, we derive Brown's upper bound for $\omega(\operatorname{Sym}(n))$ mentioned in Sect. 1.3, see [4, Theorem 1 (1)].

Proposition 3.2 There exists a constant $b$, which does not depend on $n$, such that $\omega(\operatorname{Sym}(n)) \leq b(\log \log n)(n-2) !$.

Proof By [8, Theorem 2], the number of maximal abelian subgroups of $\operatorname{Sym}(n)$ is at most $b(\log \log n)(n-2)$ ! for some constant $b$ not depending on $n$. Thus the proposition follows from Lemma 3.1.

Unfortunately, there is no natural description (as in $\operatorname{Sym}(n)$ ) for the maximal abelian subgroups of $\mathrm{GL}_{n}(q)$. So, it looks particularly difficult to give an upper bound for the number of all maximal abelian subgroups of $\mathrm{GL}_{n}(q)$. (For some results on the number of maximal abelian subgroups with trivial unipotent radical in Chevalley groups, we refer the reader to [19].) We overcome this difficulty by focusing only on the subfamily $\mathcal{A}_{n}(q)$ of abelian subgroups defined in Definition 1.5, which is large enough to cover all the group elements, as will be proved in Proposition 5.1. This leads to an upper bound for $\omega\left(\mathrm{GL}_{n}(q)\right)$. For $q>n$, we construct a pairwise noncommuting set of size $\left|\mathcal{A}_{n}(q)\right|$, and so we obtain an explicit formula for $\omega\left(\mathrm{GL}_{n}(q)\right)$.

Remark 3.3 We note that, for general $q$ and $n$, centralisers of cyclic matrices do not cover all the elements in $\mathrm{GL}_{n}(q)$. Here we simply give an example for $\mathrm{GL}_{4}(2)$. Consider the matrix

$$
x=\left(\begin{array}{llll}
1 & 0 & 0 & 0 \\
0 & 1 & 1 & 0 \\
0 & 0 & 1 & 1 \\
0 & 0 & 0 & 1
\end{array}\right)
$$

With an easy computation, it is easy to check that

$$
C=C_{\mathrm{GL}_{4}(2)}(x)=\left\{\left(\begin{array}{cccc}
1 & 0 & 0 & a \\
b & 1 & c & d \\
0 & 0 & 1 & c \\
0 & 0 & 0 & 1
\end{array}\right) \mid a, b, c, d \in \mathbb{F}_{2}\right\}
$$

and that $C$ has order 16. In particular, $C$ consists of unipotent elements. A unipotent matrix $u$ is cyclic if and only if $u$ has minimum polynomial $(t-1)^{4}$, that is, $u-1$ has rank 3. Now, it is easy to see that if $c \in C$, then $c-1$ has rank at most 2 . Therefore, $C$ contains no cyclic matrix, and hence $x$ is not contained in the centraliser of a cyclic matrix.

A similar example can be constructed for every $q>2$. Namely, consider the matrix

$$
x=\left(\begin{array}{cc}
D & 0 \\
0 & U
\end{array}\right)
$$


in $\operatorname{GL}_{2 q-1}(q)$, where $D$ is a $(q-1) \times(q-1)$-diagonal matrix with distinct eigenvalues, and $U$ is a $(q \times q)$-cyclic matrix with minimum polynomial $(t-1)^{q}$ (that is, a regular unipotent element of $\mathrm{GL}_{q}(q)$ ). It is possible to show that $x$ is not contained in the centraliser of a cyclic matrix of $\mathrm{GL}_{2 q-1}(q)$.

\section{Conjugacy classes and centralisers in $\mathrm{GL}_{n}(q)$}

In this section, we introduce some notation and some well-known results that are going to be used throughout the rest of the paper.

Let $k$ be a field with $q$ elements, $V$ be $k^{n}$, and $k[t]$ be the polynomial ring with coefficients in $k$. Now, each element $g$ of $\operatorname{GL}_{n}(q)$ acts on the vector space $V$ and hence defines on $V$ a $k[t]$-module structure by setting $t v=g v$. We denote this $k[t]$ module by $V_{g}$. For instance, it is easy to see that $g$ is a cyclic matrix if and only if $V_{g}$ is a cyclic $k[t]$-module. Clearly, any two elements $g, h$ of $\operatorname{GL}_{n}(q)$ are conjugate if and only if $V_{g}$ and $V_{h}$ are isomorphic $k[t]$-modules.

For each element $g$ of $\mathrm{GL}_{n}(q)$, there exist unique $s, u \in \mathrm{GL}_{n}(q)$ such that $g=$ $s u=u s$, where $s$ is semisimple, and $u$ is unipotent (see [5, Sect. 1.4]). We call $s$ (respectively $u$ ) the semisimple (respectively unipotent) part of $g$.

A unipotent element $u$ of $\mathrm{GL}_{n}(q)$ is said to be a regular unipotent element if $u-1$ has rank $n-1$, that is $u$ has minimum polynomial $(t-1)^{n}$, and so $u$ is a cyclic matrix. In particular, regular unipotent elements of $\mathrm{GL}_{n}(q)$ form a $\mathrm{GL}_{n}(q)$-conjugacy class. In the following lemma, we collect some well-known information on the centraliser and normaliser of a regular unipotent element.

Lemma 4.1 Let $u$ be a regular unipotent element of $\mathrm{GL}_{n}(q)$ for $n \geq 2$. The group $C=C_{\mathrm{GL}_{n}(q)}(u)$ is abelian of order $\left(1-q^{-1}\right) q^{n}$, and $N_{\mathrm{GL}_{n}(q)}(C)$ has order $(1-$ $\left.q^{-1}\right)^{2} q^{2 n-1}$.

Proof Set $v=u-1$. Since $u$ is a regular unipotent element, the element $v$ is a nilpotent matrix of rank $n-1$ with minimal polynomial $t^{n}$. Also, $C_{\mathrm{GL}_{n}(q)}(u)=$ $C_{\mathrm{GL}_{n}(q)}(v)$. The elements centralising $v$ are the isomorphisms of the $k[t]$-module $V_{v}$. Since $V_{v} \cong k[t] /\left(t^{n}\right)$ is a uniserial module, it is readily seen (see for example [14, p. 265]) that $\operatorname{End}_{k[t]}\left(V_{v}\right)$ is a polynomial ring in $v$ isomorphic to $k[t] /\left(t^{n}\right)$. Therefore, $\operatorname{End}_{k[t]}\left(V_{v}\right)=\left\langle 1, v, \ldots, v^{n-1}\right\rangle$ is abelian. Since the ideals of $k[t] /\left(t^{n}\right)$ are in one-to-one correspondence with the ideals of $k[t]$ that contain $\left(t^{n}\right)$ and $(t)$ is the unique maximal ideal containing $\left(t^{n}\right)$, it follows that $\operatorname{End}_{k[t]}\left(V_{v}\right)$ is a local ring with maximal ideal $(v)=\left\langle v, \ldots, v^{n-1}\right\rangle$ and every element of $(v)$ is nilpotent. In particular, the element $x=\sum_{i=0}^{n-1} a_{i} v^{i}$ of $\operatorname{End}_{k[t]}\left(V_{v}\right)$ is invertible if and only if $x \notin(v)$, that is $a_{0} \neq 0$. This shows that $C$ is abelian of order $q^{n}-q^{n-1}=\left(1-q^{-1}\right) q^{n}$.

Let $x=\sum_{i=0}^{n-1} a_{i} v^{i}$ be in $\operatorname{End}_{k[t]}\left(V_{v}\right)$. We claim that $x$ is a regular unipotent element if and only if $a_{0}=1$ and $a_{1} \neq 0$. Assume first that $x$ is a regular unipotent element. Thus, $x-1$ is a nilpotent element with minimum polynomial $t^{n}$. Now, $x-1$ is nilpotent if and only if $a_{0}-1=0$, that is $a_{0}=1$. Also, as $\left(v^{2}\right)^{m}=0$ for every $m \geq n / 2$, we obtain that $x-1$ is not a multiple of $v^{2}$, that is $a_{1} \neq 0$. Conversely, assume that $a_{0}=1$ and $a_{1} \neq 0$. In particular, $x-1=v y$, where by the previous 
paragraph $y$ is an invertible element of $\operatorname{End}_{k[t]}\left(V_{v}\right)$. So, $(x-1)^{n-1}=v^{n-1} y^{n-1} \neq 0$, and $x-1$ has minimum polynomial $v^{n-1}$. Thus $x$ is a regular unipotent element. This yields that $C$ contains $q^{n-1}-q^{n-2}=\left(1-q^{-1}\right) q^{n-1}$ regular unipotent elements. Since the regular unipotent elements form a $\mathrm{GL}_{n}(q)$-conjugacy class, $C$ contains $\left(1-q^{-1}\right) q^{n-1}$ regular unipotent elements and $C=C_{\mathrm{GL}_{n}(q)}\left(u^{\prime}\right)$ for each regular unipotent element $u^{\prime} \in C$, we have that $\left|N_{\mathrm{GL}_{n}(q)}(C)\right| /|C|=\left(1-q^{-1}\right) q^{n-1}$ and $\left|N_{\mathrm{GL}_{n}(q)}(C)\right|=\left(1-q^{-1}\right)^{2} q^{2 n-1}$.

Let $d, m \geq 1$ be integers such that $n=d m$, and $E$ be a field extension over $k$ of degree $d$. As $E$ is a $k$-vector space of dimension $d$ and $d$ divides $n$, we have that $k^{n}$ is isomorphic to $E^{m}$ as $k$-vector spaces. Under this isomorphism, the group $\operatorname{GL}_{m}\left(q^{d}\right)$ is embedded into $\mathrm{GL}_{n}(q)$, and we still denote the image by $\mathrm{GL}_{m}\left(q^{d}\right)$. This does not cause any confusion because all fields of order $q^{d}$ are isomorphic, and therefore different embeddings give rise to subgroups which are conjugate.

We recall that, given a group $G$, a $G$-module $V$ is said to be indecomposable if $V \neq 0$ and if it is impossible to express $V$ as a direct sum of two non-trivial $G$ submodules. In the next lemma we determine the elements $g$ of $\mathrm{GL}_{n}(q)$ such that $V_{g}$ is indecomposable.

Lemma 4.2 Let $g \in \mathrm{GL}_{n}(q)$ be such that $V_{g}$ is an indecomposable $k[t]$-module and $g$ has the semisimple part $s$ and the unipotent part $u$. Then $g$ is a cyclic matrix, and there exists an irreducible polynomial $f$ of degree $d$ such that the minimum polynomial of $g$ equals $f^{m}$ and $d m=n$. Replacing $g$ by a conjugate if necessary, we obtain that $g \in \mathrm{GL}_{m}\left(q^{d}\right)$, $s$ is a scalar matrix in $\mathrm{GL}_{m}\left(q^{d}\right)$, corresponding to a generator of $\mathbb{F}_{q^{d}}$ as a field, while $u$ is a regular unipotent element of $\mathrm{GL}_{m}\left(q^{d}\right)$. In particular, $C_{\mathrm{GL}_{n}(q)}(\mathrm{g})$ is abelian of order $\left(1-q^{-d}\right) q^{d m}$.

Proof Since $k[t]$ is a principal ideal domain, we have that the $k[t]$-module $V_{g}$ is a direct sum of cyclic modules of the form $k[t] /\left(f^{m}\right)$, where $f$ is a monic irreducible polynomial of $k[t]$ and $m \geq 1$. As $V_{g}$ is indecomposable, we obtain that $V_{g} \cong k[t] /\left(f^{m}\right)$ for some irreducible polynomial $f=t^{d}-\sum_{i=1}^{d} a_{i} t^{i-1}$ of degree $d$, and $n=d m$. Let $J(f)$ denote the companion matrix for the polynomial $f$

$$
J(f)=\left(\begin{array}{ccccc}
0 & 1 & 0 & \cdots & 0 \\
0 & 0 & 1 & \cdots & 0 \\
\cdots & & & & \cdots \\
0 & 0 & 0 & \cdots & 1 \\
a_{1} & a_{2} & & \cdots & a_{d}
\end{array}\right),
$$

and let

$$
J_{m}(f)=\left(\begin{array}{ccccc}
J(f) & I_{d} & 0 & \ldots & 0 \\
0 & J(f) & I_{d} & \ddots & \vdots \\
\vdots & \ddots & \ddots & \ddots & \\
\vdots & & \ddots & J(f) & I_{d} \\
0 & \ldots & \ldots & 0 & J(f)
\end{array}\right)
$$


with $m$ diagonal blocks $J(f)$. By construction the characteristic polynomial of the block matrix $J_{m}(f)$ equals $f^{m}$. Also, [11, Example 1, page 140] shows that $f^{m}$ is the minimum polynomial of $J_{m}(f)$. Therefore $J_{m}(f)$ is a cyclic matrix. As $V_{g}$ and $V_{J_{m}(f)}$ are $k[t]$-modules isomorphic to $k[t] /\left(f^{m}\right)$, we obtain that $g$ is conjugate to $J_{m}(f)$ and so $g$ is a cyclic matrix with minimum polynomial $f^{m}$. Thus we may assume that $g=J_{m}(f)$. So, $s$ is obtained from $J_{m}(f)$ by replacing the $d \times d$-identity matrix $I_{d}$ with the $d \times d$-zero matrix 0 . Similarly, $u$ is obtained from $J_{m}(f)$ by replacing the $d \times d$-matrix $J(f)$ with the $d \times d$-identity matrix $I_{d}$.

Now, the centraliser of the cyclic matrix $J(f)$ in the algebra of $d \times d$-matrices over $k$ is a polynomial algebra isomorphic to $k[t] /(f)$. Since $f$ is irreducible, $k[t] /(f)$ is a field of size $q^{d}$. Hence $C_{\mathrm{GL}_{d}(q)}(J(q))$ is a cyclic group of order $q^{d}-1$ isomorphic to the multiplicative group of a field of size $q^{d}$, and since $f$ is irreducible, $J(f)$ corresponds to a generator in this field. Under this identification, $J_{m}(f)$ is an element of $\mathrm{GL}_{m}\left(q^{d}\right), s$ is a scalar matrix corresponding to a generator of $\mathbb{F}_{q^{d}}$, and $u$ is a regular unipotent matrix. The rest of the lemma follows from Lemma 4.1.

Corollary 4.3 Let $g_{1}$ and $g_{2}$ be in $\mathrm{GL}_{n}(q)$ such that $V_{g_{1}}$ and $V_{g_{2}}$ are indecomposable $k[t]$-modules. Set $C_{g_{i}}=C_{\mathrm{GL}_{n}(q)}\left(g_{i}\right)$ for $i=1,2$. The following are equivalent:

(i) $C_{g_{1}}$ is conjugate to $C_{g_{2}}$.

(ii) $g_{1}$ and $g_{2}$ have minimum polynomials $f_{g_{1}}^{m}$ and $f_{g_{2}}^{m}$ for some irreducible polynomials $f_{g_{1}}, f_{g_{2}}$ of degree $d$ with $d m=n$.

(iii) the $C_{g_{1}}$-module $V$ is isomorphic to the $C_{g_{2}}$-module $V$.

Proof By Lemma 4.2, $g_{i}$ is a cyclic matrix with minimum polynomial $f_{g_{i}}^{m_{g_{i}}}$ for some irreducible polynomial $f_{g_{i}}$ of degree $d_{g_{i}}$ with $d_{g_{i}} m_{g_{i}}=n$ (for $i=1,2$ ). Assume that $C_{g_{1}}$ is conjugate to $C_{g_{2}}$. By Lemma $4.2,\left|C_{g_{1}}\right|=\left(1-q^{-d_{g_{1}}}\right) q^{d_{g_{1}} m_{g_{1}}}$ and $\left|C_{g_{2}}\right|=$ $\left(1-q^{-d_{g_{2}}}\right) q^{d_{g_{2}} m_{g_{2}}}$. As $\left|C_{g_{1}}\right|=\left|C_{g_{2}}\right|$, we have $d_{g_{1}}=d_{g_{2}}$ and $m_{g_{1}}=m_{g_{2}}$. Thus Part (i) implies Part (ii).

Assume Part (ii). Let $s_{1}$ and $s_{2}$ be the semisimple parts of $g_{1}$ and $g_{2}$, respectively. Similarly, let $u_{1}$ and $u_{2}$ be the unipotent parts of $g_{1}$ and $g_{2}$, respectively. By Lemma 4.2, replacing $g_{1}$ and $g_{2}$ by a conjugate if necessary, we may assume that $g_{1}, g_{2} \in \mathrm{GL}_{m}\left(q^{d}\right), s_{1}$ and $s_{2}$ are scalar matrices corresponding to generators of $\mathbb{F}_{q}$, and $u_{1}, u_{2}$ are regular unipotent elements of $\operatorname{GL}_{m}\left(q^{d}\right)$. Therefore $C_{g_{i}}=C_{\mathrm{GL}_{n}(q)}\left(g_{i}\right)=C_{\mathrm{GL}_{m}\left(q^{d}\right)}\left(g_{i}\right)=C_{\mathrm{GL}_{m}\left(q^{d}\right)}\left(u_{i}\right)$ for $i=1$, 2. Since regular unipotent elements form a $\mathrm{GL}_{m}\left(q^{d}\right)$-conjugacy class, we obtain that $u_{1}$ is conjugate to $u_{2}$ in $\mathrm{GL}_{m}\left(q^{d}\right)$; so $C_{g_{1}}=C_{\mathrm{GL}_{m}\left(q^{d}\right)}\left(u_{1}\right)$ is conjugate to $C_{g_{2}}=C_{\mathrm{GL}_{m}\left(q^{d}\right)}\left(u_{2}\right)$, and Part (i) follows.

If $C_{g_{1}}$ is conjugate in $\mathrm{GL}_{n}(q)$ to $C_{g_{2}}$, then the $C_{g_{1}}$-module $V$ is isomorphic to the $C_{g_{2}}$-module $V$. Thence Part (i) implies Part (iii).

Conversely, if the $C_{g_{1}}$-module $V$ is isomorphic to the $C_{g_{2}}$-module $V$, then there exist a group isomorphism $\varphi: C_{g_{1}} \rightarrow C_{g_{2}}$ and a $k$-vector space isomorphism $\psi$ : $V \rightarrow V$ such that $(v g) \psi=(v \psi)\left(g^{\varphi}\right)$ for every $v \in V$ and $g \in C_{g_{1}}$. This yields $g^{\varphi}=$ $\psi^{-1} g \psi$ for every $g \in C_{g_{1}}$. Thence $C_{g_{1}}$ is conjugate to $C_{g_{2}}$ in $\operatorname{GL}_{n}(q)$, and Part (i) follows. 
The set of abelian subgroups $\left\{C_{\mathrm{GL}_{n}(q)}(g) \mid V_{g}\right.$ indecomposable $\}$ of $\mathrm{GL}_{n}(q)$ plays a very important role in this paper. It is worth to point out that, by Corollary 4.3 , the conjugacy classes in this family of subgroups are in one-to-one correspondence with the ordered pairs of positive integers $(d, m)$ with $n=d m$. We denote by

$$
\left\{A_{d, m}\right\}_{d, m}
$$

a set of representatives for these conjugacy classes. In particular, for $m=1$, the group $A_{d, 1}$ is a cyclic group, and actually $A_{d, 1}$ is a maximal non-split torus of order $q^{d}-1$ in $\mathrm{GL}_{d}(q)$, usually called a Singer cycle.

Lemma 4.4 Let $d, m \geq 1$ be such that $n=d m$. The group $A_{d, m}$ is a maximal abelian subgroup of $\mathrm{GL}_{n}(q)$ and

$$
\left|N_{\mathrm{GL}_{n}(q)}\left(A_{d, m}\right)\right|= \begin{cases}d\left(1-q^{-d}\right)^{2} q^{2 d m-d} & \text { if } m>1 \\ d\left(1-q^{-d}\right) q^{d} & \text { if } m=1 .\end{cases}
$$

Proof By the definition of $A_{d, m}$, there exists an element $g=s u$ of $A_{d, m}$ such that $A_{d, m}=C_{\mathrm{GL}_{n}(q)}(g)$, where $s$ is the semisimple part of $g$, and $u$ is the unipotent part of $g$. By Lemma 4.2, we may choose $A_{d, m}$ such that $A_{d, m} \subseteq \mathrm{GL}_{m}\left(q^{d}\right)$, and we may assume that $s$ is a scalar matrix of $\operatorname{GL}_{m}\left(q^{d}\right)$ of order $q^{d}-1$.

By Lemma 4.2, $A_{d, m}$ is abelian. Let $A$ be an abelian subgroup of $\operatorname{GL}_{n}(q)$ containing $A_{d, m}$, and $x$ be in $A$. Since $A$ is abelian, $x$ commutes with $g$, and so $x \in A_{d, m}$. This yields that $A_{d, m}$ is a maximal abelian subgroup of $\operatorname{GL}_{n}(q)$.

Let $N$ be the normaliser in $\mathrm{GL}_{n}(q)$ of $A_{d, m}$, and let $x \in N$. Since $\langle s\rangle$ is a normal Hall subgroup of $A_{d, m}$, it follows that $x$ normalises $\langle s\rangle$. Thus $x$ normalises the subgroup of scalar matrices of $\mathrm{GL}_{m}\left(q^{d}\right)$, and so $x$ induces a Galois automorphism on this subgroup, viewed as the multiplicative group of the field of order $q^{d}$. Since the full Galois group over $k[s]$ is induced, it follows that $\left|N: N \cap \mathrm{GL}_{m}\left(q^{d}\right)\right|=d$. If $m=1$, then $\operatorname{GL}_{1}\left(q^{d}\right)=A_{d, 1}$ and $|N|=d\left(q^{d}-1\right)$. If $m>1$, then Lemma 4.1 yields that $N \cap \mathrm{GL}_{m}\left(q^{d}\right)$ has order $\left(1-q^{-d}\right)^{2} q^{2 d m-d}$.

\section{The family $\mathcal{A}_{n}(q)$ and the upper bound for $\omega\left(\operatorname{GL}_{n}(q)\right)$}

We now study the family $\mathcal{A}_{n}(q)$ of abelian subgroups of $\mathrm{GL}_{n}(q)$ introduced in Definition 1.5 and obtain an upper bound on $\omega\left(\mathrm{GL}_{n}(q)\right)$. For convenience, we state Definition 1.5 again.

Definition 1.5 Let $\mathcal{A}_{n}(q)$ be the set of abelian subgroups $A$ of $\mathrm{GL}_{n}(q)$ such that the $A$-module $V$ has a decomposition $V_{1} \oplus \cdots \oplus V_{r}$ into indecomposable $A$-modules satisfying the following properties:

(i) $A=A_{1} \times \cdots \times A_{r}$, where $A_{i} \subseteq \mathrm{GL}\left(V_{i}\right)$.

(ii) for $i=1, \ldots, r$, we have $A_{i}=C_{\mathrm{GL}\left(V_{i}\right)}\left(a_{i}\right)$ for some element $a_{i} \in \mathrm{GL}\left(V_{i}\right)$ such that $\left(V_{i}\right)_{a_{i}}$ is an indecomposable $k[t]$-module. 
We show in Proposition 5.7 that, for $q>2$, the elements of $\mathcal{A}_{n}(q)$ are maximal abelian subgroup of $\mathrm{GL}_{n}(q)$. From the definition of $\mathcal{A}_{n}(q)$ we get at once Theorem 1.4(a).

Proposition 5.1 $\mathrm{GL}_{n}(q)=\bigcup_{A \in \mathcal{A}_{n}(q)} A$.

Proof Given $x$ in $\mathrm{GL}_{n}(q)$, consider a decomposition of $V_{x}=V_{1} \oplus \cdots \oplus V_{r}$ into indecomposable $k[t]$-modules. The action of $x$ on $V_{i}$ is given by some element $a_{i} \in$ $\mathrm{GL}\left(V_{i}\right)$. By Lemma 4.2, $A_{i}=C_{\mathrm{GL}\left(V_{i}\right)}\left(a_{i}\right)$ is abelian. Now, $x \in A=A_{1} \times \cdots \times A_{r}$ and $A \in \mathcal{A}_{n}(q)$.

The following definition is necessary in order to have a natural set of labels for the elements in $\mathcal{A}_{n}(q)$ (see Lemma 5.3).

Definition 5.2 We denote by $\Phi$ the set of maps from $\mathbb{N} \times \mathbb{N}=\{(d, m) \mid d, m \geq 1\}$ to $\mathbb{N}$. Also, we write $\Phi_{n}$ for the subset of $\Phi$ containing the functions $\mu: \mathbb{N} \times \mathbb{N} \rightarrow \mathbb{N}$ such that $n=\sum_{d, m \geq 1} d m \mu(d, m)$.

For instance, $\Phi_{1}$ contains only one element, namely the function $\mu$ defined by $\mu(1,1)=1$ and $\mu(d, m)=0$ for $m>1$ or $d>1$.

Lemma 5.3 The conjugacy classes of subgroups in $\mathcal{A}_{n}(q)$ are in one-to-one correspondence with the elements of $\Phi_{n}$.

Proof We define a bijection $\theta$ from $\Phi_{n}$ to the set of conjugacy classes of subgroups in $\mathcal{A}_{n}(q)$. Let $\mu$ be in $\Phi_{n}$. Let $e_{1}, \ldots, e_{n}$ be a basis of the $k$-vector space $V$. Since $n=\sum_{d, m \geq 1} d m \mu(d, m)$, we can partition the set $\{1, \ldots, n\}$ in subsets $Y_{d, m}$ of size $d m \mu(d, m)$ for all $d, m \geq 1$. So $\{1, \ldots, n\}=\bigcup_{d, m \geq 1} Y_{d, m}$. Given $d, m \geq 1$, the set $Y_{d, m}$ has size $d m \mu(d, m)$ and so can be partitioned in $\mu(d, m)$ subsets $Y_{d, m, i}$ of size $d m$ for each $1 \leq i \leq \mu(d, m)$. So, $Y_{d, m}=\bigcup_{1 \leq i \leq \mu(d, m)} Y_{d, m, i}$. For all $d, m \geq 1$ and $i$ with $1 \leq i \leq \mu(d, m)$, let $W_{d, m, i}=\left\langle e_{y} \mid y \in Y_{d, m, i}\right\rangle$. By construction, we have $V=\bigoplus_{d, m \geq 1}\left(\bigoplus_{1 \leq i \leq \mu(d, m)} W_{d, m, i}\right)$.

Consider $A_{d, m}^{(i)} \leq \operatorname{GL}\left(W_{d, m, i}\right)$ with $A_{d, m}^{(i)}=A_{d, m}$ as in (4.1), and set $A=$ $\prod_{d, m \geq 1} \prod_{1 \leq i \leq \mu(d, m)} A_{d, m}^{(i)}$. By construction, $A \in \mathcal{A}_{n}(q)$. Define $\theta(\mu)$ to be the conjugacy class containing $A$. Now, let $V=\bigoplus_{k=1}^{s} M_{k}$ be another decomposition of $V$ into a direct sum of non-zero indecomposable $A$-submodules. By the Krull-Schmidt theorem [7, Theorem 14.5], there exists a bijective function $f$ between the set of indices $\{(d, m, i) \mid 1 \leq i \leq \mu(d, m)\}$ and $\{1, \ldots, s\}$ such that $W_{d, m, i} \cong M_{f(d, m, i)}$. Thus, Corollary 4.3 yields that $\mu$ is uniquely determined from the conjugacy class of $A$ in $\mathrm{GL}_{n}(q)$, that is, $\theta$ is injective.

The map $\theta$ is surjective by the definition of $\mathcal{A}_{n}(q)$.

Given $\mu \in \Phi_{n}$, we denote by

$$
A_{\mu}
$$


a representative of the conjugacy class in $\mathcal{A}_{n}(q)$ corresponding to $\mu$ in $\Phi_{n}$. We note that if $\mu(d, m)=0$ for $m>1$, then $A_{\mu}$ is a torus in $\operatorname{GL}_{n}(q)$. In particular, every maximal torus of $\mathrm{GL}_{n}(q)$ is a member of $\mathcal{A}_{n}(q)$.

Before proving the main result of this paper, we need first a definition and then some preliminary lemmas.

Definition 5.4 Let $\mu$ be in $\Phi_{n}$ and $q=2$. We say that $A_{\mu}$ has cyclic unipotent summand if $\mu(1, x)=0$ for all but at most one value of $x$, and if $\mu(1, x) \neq 0$ for $x=m$, say, then $\mu(1, m)=1$. In particular, by Definition 1.5 and Lemma 4.2, $V$ has at most one indecomposable $A_{\mu}$-invariant summand $W$ such that the action of $A_{\mu}$ on $W$ is given by the centraliser of a regular unipotent matrix (which is a cyclic matrix).

Lemma 5.5 Let $\mu$ be in $\Phi_{n}$. The decomposition of $V$ as direct sum of indecomposable $A_{\mu}$-modules is unique up to permutation of the summands if and only if either $q \geq 3$, or $q=2$ and $A_{\mu}$ has cyclic unipotent summand.

\section{Proof Let}

$$
V=\bigoplus_{\substack{d, m \geq 1, 1 \leq i \leq \mu(d, m)}} V_{d, m}^{(i)}
$$

be an $A_{\mu}$-invariant direct decomposition in indecomposable modules labelled so that $\operatorname{dim} V_{d, m}^{(i)}=d m$ (see Lemma 5.3). By Definition 1.5(i), we have $A_{\mu}=$ $\prod_{d, m \geq 1} \prod_{1 \leq i \leq \mu(d, m)} A_{d, m}^{(i)}$, where $A_{d, m}^{(i)} \subseteq \operatorname{GL}\left(V_{d, m}^{(i)}\right)$.

Assume that $q \geq 3$, or $q=2$ and $A_{\mu}$ has cyclic unipotent summand. We show that the decomposition of $V$ as direct sum of indecomposable $A_{\mu}$-modules is unique, up to permutation of the summands. Let $W$ be an indecomposable $A_{\mu}$-invariant summand of $V$. Now, the $A_{\mu}$-module $W$ is cyclic, that is, there exists $v \in W$ such that $W=$ $\langle v\rangle_{A_{\mu}}$ (where $\langle v\rangle_{A_{\mu}}=\left\langle v a \mid a \in A_{\mu}\right\rangle$ ). Write $v=\sum_{d, m \geq 1} \sum_{1 \leq i \leq \mu(d, m)} v_{d, m}^{(i)}$, with $v_{d, m}^{(i)} \in V_{d, m}^{(i)}$. We claim that

$$
W=\bigoplus_{d, m \geq 1} \bigoplus_{1 \leq i \leq \mu(d, m)}\left\langle v_{d, m}^{(i)}\right\rangle_{A_{\mu}} .
$$

The $A_{\mu}$-module $W$ is generated by $v a=\sum_{d, m, i} v_{d, m}^{(i)} a$ (for $a \in A_{\mu}$ ), where $v_{d, m}^{(i)} a \in$ $\left\langle v_{d, m}^{(i)}\right\rangle_{A_{\mu}}$. Therefore, $W \subseteq \sum_{d, m, i}\left\langle v_{d, m}^{(i)}\right\rangle_{A_{\mu}}$. Conversely, as $\left\langle v_{d, m}^{(i)}\right\rangle_{A_{\mu}} \cap\left\langle v_{d^{\prime}, m^{\prime}}^{\left(i^{\prime}\right)}\right\rangle_{A_{\mu}} \subseteq$ $V_{d, m}^{(i)} \cap V_{d^{\prime}, m^{\prime}}^{\left(i^{\prime}\right)}=0$ for $(d, m, i) \neq\left(d^{\prime}, m^{\prime}, i^{\prime}\right)$, it suffices to prove that $v_{d, m}^{(i)} \in W$ for all $d, m, i$. By Definition 1.5(ii) and Lemma 4.2, the group $A_{d, m}^{(i)}$ contains a scalar matrix $s_{d, m}^{(i)}$ of $\mathrm{GL}_{m}\left(q^{d}\right)$ corresponding to a generator of $\mathbb{F}_{q^{d}}$ if $d \geq 2$, and to a primitive element of $\mathbb{F}_{q}$ if $d=1$. In particular, $s_{d, m}^{(i)} \neq 1$ if $(q, d) \neq(2,1)$.

Assume first that $(q, d) \neq(2,1)$. Since $s_{d, m}^{(i)}$ acts as the identity matrix on $V_{d^{\prime}, m^{\prime}}^{\left(i^{\prime}\right)}$ (for $\left.\left(d^{\prime}, m^{\prime}, i^{\prime}\right) \neq(d, m, i)\right)$, we get $v s_{d, m}^{(i)}=\sum_{\left(d^{\prime}, m^{\prime}, i^{\prime}\right) \neq(d, m, i)} v_{d^{\prime}, m^{\prime}}^{\left(i^{\prime}\right)}+v_{d, m}^{(i)} s_{d, m}^{(i)}$. 
Therefore, $v_{d, m}^{(i)}\left(s_{d, m}^{(i)}-1\right)=v s_{d, m}^{(i)}-v \in W$. As $s_{d, m}^{(i)}$ acts as a non-identity scalar matrix on $V_{d, m}^{(i)}$, we obtain $v_{d, m}^{(i)} \in W$. This yields that if $(q, d) \neq(2,1)$, then $v_{d, m}^{(i)}$ lies in $W$ for every $m$ and $i$. By hypothesis on $q$ and $A_{\mu}$ and by Definition 5.4, we obtain that all but possibly one summand of $v$ lies in $W$. The exceptional case $(q, d)=(2,1)$ occurs only if $q=2$ and $v_{1, m}^{(1)}$ is the only summand of $v$ that is not covered by the argument in this paragraph; since $v \in W$, we get also in this case that $v_{1, m}^{(1)} \in W$ and hence that every summand of $v$ lies in $W$. Our claim is now proved.

Since $W$ is indecomposable, from (5.2) we have $W=\left\langle v_{d, m}^{(i)}\right\rangle_{A_{\mu}} \subseteq V_{d, m}^{(i)}$ for some $d, m, i$. Since $W$ is an $A_{\mu}$-invariant summand and $V_{d, m}^{(i)}$ is indecomposable, $W=$ $V_{d, m}^{(i)}$. As $W$ is an arbitrary indecomposable summand of $V$, we get that $\left\{V_{d, m}^{(i)}\right\}_{d, m, i}$ are the only indecomposable summands of $V$ and the decomposition is unique.

Conversely, assume that $q=2$ and $A_{\mu}$ does not have a cyclic unipotent summand, that is, $\mu(1, m) \geq 2$ for some $m$, or $\mu\left(1, m_{1}\right)=\mu\left(1, m_{2}\right)=1$ with $m_{1} \neq$ $m_{2}$. Let $V_{1}$ and $V_{2}$ be two distinct $A_{\mu}$-invariant indecomposable direct summands of $V$ isomorphic to $V_{1, m}$ (if $\mu(1, m) \geq 2$ ) or isomorphic to $V_{1, m_{1}}$ and $V_{1, m_{2}}$ (if $\left.\mu\left(1, m_{1}\right)=\mu\left(1, m_{2}\right)=1\right)$. By Lemma 4.2, (4.1) and Definition 1.5, $V_{i}$ is an $A_{i}$ module, where $A_{i}=C_{\mathrm{GL}\left(V_{i}\right)}\left(u_{i}\right)$, and $u_{i}$ is a regular unipotent matrix of $\operatorname{GL}\left(V_{i}\right)$. Let $v_{1,1}, \ldots, v_{1, r_{1}}$ (respectively, $v_{2,1}, \ldots, v_{2, r_{2}}$ ) be a $k$-basis of $V_{1}$ (respectively, $V_{2}$ ) such that $v_{i, j}^{u_{i}}=v_{i, j}+v_{i, j-1}$ (for $1<j \leq r_{i}$ ) and $v_{i, 1}^{u_{i}}=v_{i, 1}$ for $i=1,2$. Define $V_{2}^{\prime}=\left\langle v_{2,1}, \ldots, v_{2, r_{2}-1}, v_{1,1}+v_{2, r_{2}}\right\rangle$. Clearly, $V_{1} \oplus V_{2}=V_{1} \oplus V_{2}^{\prime}$. We claim that $V_{2}^{\prime}$ is an indecomposable $A_{\mu}$-invariant summand of $V$. Since $u_{2}$ is a cyclic matrix, $\operatorname{End}_{k\left\langle u_{2}\right\rangle}\left(V_{2}\right)$ is a polynomial algebra in $u_{2}$. Therefore, in order to show that $V_{2}^{\prime}$ is an $A_{\mu}$-invariant summand of $V$, it suffices to show that $V_{2}^{\prime}$ is $\left\langle u_{2}\right\rangle$-invariant, which is clear from the definition of $V_{2}^{\prime}$ and from the action of $u_{2}$ on $V_{2}$.

As $V_{2^{\prime}} \subseteq V_{1} \oplus V_{2}, V_{2}^{\prime} \neq V_{1}$ and $V_{2}^{\prime} \neq V_{2}$, we obtain that the decomposition of $V$ as a direct sum of indecomposable $A_{\mu}$-modules is not unique.

We give a definition which is needed in Proposition 5.7 and in Sect. 6.

Definition 5.6 Let $\mu$ be in $\Phi_{n}$, and $V=V_{1} \oplus \cdots \oplus V_{r}$ be an $A_{\mu}$-invariant decomposition of $V$ into indecomposable modules. We write $\operatorname{Stab}(V, \mu)$ for the subgroup of $\mathrm{GL}_{n}(q)$ preserving the direct decomposition $V_{1} \oplus \cdots \oplus V_{r}$ of $V$, that is, $\operatorname{Stab}(V, \mu)=\left\{g \in \mathrm{GL}_{n}(q) \mid V_{i}^{g} \in\left\{V_{1}, \ldots, V_{r}\right\}\right.$ for every $\left.i\right\}$.

We start by computing the size of the normaliser of a subgroup $A_{\mu}$ and by proving that, if $q \geq 3$, or $q=2$ and $A_{\mu}$ has cyclic unipotent summand, then $A_{\mu}$ is a maximal abelian subgroup of $\mathrm{GL}_{n}(q)$, for $\mu \in \Phi$.

Proposition 5.7 Let $\mu$ be in $\Phi_{n}$. Then $\left|N_{\mathrm{GL}_{n}(q)}\left(A_{\mu}\right) \cap \operatorname{Stab}(V, \mu)\right|$ equals

$$
\begin{aligned}
& \left(\prod_{d \geq 1}\left(d\left(1-q^{-d}\right) q^{d}\right)^{\mu(d, 1)} \mu(d, 1) !\right) \\
& \quad \times\left(\prod_{d \geq 1, m \geq 2}\left(d\left(1-q^{-d}\right)^{2} q^{2 d m-d}\right)^{\mu(d, m)} \mu(d, m) !\right) .
\end{aligned}
$$


If either $q \geq 3$, or $q=2$ and $A_{\mu}$ has cyclic unipotent summand, then $N_{\mathrm{GL}_{n}(q)}\left(A_{\mu}\right) \subseteq$ $\operatorname{Stab}(V, \mu)$, and $A_{\mu}$ is a maximal abelian subgroup of $\mathrm{GL}_{n}(q)$.

Proof Let $\mu$ be in $\Phi_{n}$. Write $A_{\mu}=\prod_{d, m \geq 1} A_{d, m}^{\mu(d, m)}$, where $A_{d, m}$ is as defined in (4.1). By Definition 5.6 we have

$$
\left|N_{\mathrm{GL}_{n}(q)}\left(A_{\mu}\right) \cap \operatorname{Stab}(V, \mu)\right|=\prod_{d, m \geq 1}\left|N_{\mathrm{GL}_{d m}(q)}\left(A_{d, m}\right)\right|^{\mu(d, m)} \mu(d, m) ! .
$$

Applying Lemma 4.4, the equality in the proposition follows.

Assume that either $q \geq 3$ or that $q=2$ and $A_{\mu}$ has cyclic unipotent summand. By Lemma 5.5, every element of $\mathrm{GL}_{n}(q)$ normalising $A_{\mu}$ induces a permutation of the indecomposable $A_{\mu}$-submodules of $V$. Also, Corollary 4.3 yields that indecomposable $A_{\mu}$-submodules are isomorphic if and only if they correspond to the same $d, m$. Therefore, $N_{\mathrm{GL}_{n}(q)}\left(A_{\mu}\right) \subseteq \operatorname{Stab}(V, \mu)$. Furthermore, by Lemma $4.4, A_{d, m}$ is a maximal abelian subgroup of $\mathrm{GL}_{d m}(q)$, and so $A_{\mu}$ is a maximal abelian subgroup of $\mathrm{GL}_{n}(q)$.

Remark 5.8 The converse of the last assertion of Proposition 5.7 is also true. Indeed, if $q=2$ and $A_{\mu}$ does not have cyclic unipotent summand, then $N_{\mathrm{GL}_{n}(q)}\left(A_{\mu}\right)$ contains an element $x$ that does not lie in $\operatorname{Stab}(V, \mu)$, and $\left\langle A_{\mu}, x\right\rangle$ is abelian. Namely, in the notation of the last part of the proof of Lemma 5.5, the element $x$ can be defined to act as the identity on all summands of the decomposition $\oplus_{i} V_{i}$, except those denoted $V_{1} \oplus V_{2}$. The action of $x$ on $V_{1} \oplus V_{2}$ is given by $v_{i, j}^{x}=v_{i, j}$ except that $v_{1,1}^{x}=v_{1,1}+$ $v_{2, r_{2}}$.

Next, we prove Theorem 1.4(c).

Theorem 5.9 $\mathcal{N}_{n}(q) \subseteq \mathcal{A}_{n}(q)$ with equality if and only if $q>n$.

Proof First we show that $\mathcal{N}_{n}(q) \subseteq \mathcal{A}_{n}(q)$. Let $C$ be an element of $\mathcal{N}_{n}(q)$. By Definition 1.2, $C=C_{\mathrm{GL}_{n}(q)}(g)$ for some cyclic matrix $g \in \mathrm{GL}_{n}(q)$. Consider a decomposition of $V_{g}=V_{1} \oplus \cdots \oplus V_{r}$ into indecomposable $k[t]$-modules. The action of $g$ on $V_{i}$ is given by some element $a_{i} \in \mathrm{GL}\left(V_{i}\right)$. By Lemma 4.2, $A_{i}=C_{\mathrm{GL}\left(V_{i}\right)}\left(a_{i}\right)$ is abelian. Now, $g \in A=A_{1} \times \cdots \times A_{r}$ and $A \in \mathcal{A}_{n}(q)$. Replacing $C$ by a conjugate if necessary, we may assume $A=A_{\mu}$ for $\mu \in \Phi_{n}$. Moreover, since $g$ is cyclic, it follows that, if $q=2$, then $A$ has cyclic unipotent summand. So, by Proposition 5.7, $A$ is a maximal abelian subgroup. As $g \in A$, we have $A \subseteq C_{\mathrm{GL}_{n}(q)}(g)=C$. Since $C$ is abelian, we have $A=C$ and $C \in \mathcal{A}_{n}(q)$.

Finally, in the rest of the proof we show that $\mathcal{N}_{n}(q)=\mathcal{A}_{n}(q)$ if and only if $q>n$.

Assume that $q>n$. As $\mathcal{N}_{n}(q) \subseteq \mathcal{A}_{n}(q)$, by Lemma 5.3, it suffices to prove that for every $\mu \in \Phi_{n}$, there exists a cyclic matrix $g_{\mu} \in A_{\mu}$ such that $A_{\mu}=C_{\mathrm{GL}_{n}(q)}\left(g_{\mu}\right)$. For all $d, m \geq 1$ such that $d m \leq n$, let $f_{d, m, 1}, \ldots, f_{d, m, \mu(d, m)}$ be irreducible polynomials of degree $d$. Note that since $q>n$, we may choose $f_{d, m, i}$ so that the polynomials 
$\left(f_{d, m, i}\right)_{d, m, i}$ are pairwise distinct. Let

$$
V=\bigoplus_{\substack{d, m \geq 1, 1 \leq i \leq \mu(d, m)}} V_{d, m}^{(i)}
$$

be an $A_{\mu}$-invariant direct decomposition in indecomposable modules labelled so that $\operatorname{dim} V_{d, m}^{(i)}=d m$ (see Lemmas 5.3). By Definition 1.5(i), we have $A_{\mu}=\prod_{d, m, i} A_{d, m}^{(i)}$, where $A_{d, m}^{(i)} \subseteq \mathrm{GL}\left(V_{d, m}^{(i)}\right)$. Also, by Definition 1.5(ii), $A_{d, m}^{(i)}=C_{\mathrm{GL}\left(V_{d, m}^{(i)}\right)}\left(h_{d, m}^{(i)}\right)$ for some element $h_{d, m}^{(i)} \in \operatorname{GL}\left(V_{d, m}^{(i)}\right)$ such that $V_{d, m}^{(i)}$ is an indecomposable $\left\langle h_{d, m}^{(i)}\right\rangle$-module. By Lemma 4.2 and Definition $4.1, h_{d, m}^{(i)}$ is a cyclic matrix with minimum polynomial $p_{d, m, i}^{m}$ for some irreducible polynomial $p_{d, m, i}$ of degree $d$. Let $g_{d, m}^{(i)} \in \operatorname{GL}\left(V_{d, m}^{(i)}\right)$ be a cyclic matrix with minimum polynomial $f_{d, m, i}^{m}$. Thence $V_{d, m}^{(i)}$ is an indecomposable $\left\langle g_{d, m}^{(i)}\right\rangle$-module. Set

$$
g_{\mu}=\bigoplus_{\substack{d, m \geq 1, 1 \leq i \leq \mu(d, m)}} g_{d, m}^{(i)}
$$

Since $f_{d, m, i}$ and $p_{d, m, i}$ have both degree $d$, by Corollary 4.3 the groups $C_{\mathrm{GL}\left(V_{d, m}^{(i)}\right)}\left(g_{d, m}^{(i)}\right)$ and $C_{\mathrm{GL}\left(V_{d, m}^{(i)}\right)}\left(h_{d, m}^{(i)}\right)=A_{d, m}^{(i)}$ are conjugate. So, by Definition 5.1 and by construction, $g_{\mu}$ is conjugate to an element in $A_{\mu}$. Hence, replacing $g_{\mu}$ by a conjugate if necessary, $g_{\mu} \in A_{\mu}$. The characteristic polynomial of $g_{\mu}$ is $\prod_{d, m, i} f_{d, m, i}^{m}$. As the polynomials $f_{d, m, i}$ are distinct, the characteristic polynomial of $g_{\mu}$ is equal to its minimum polynomial. Thence $g_{\mu}$ is a cyclic matrix. In particular, if $q=2$, then at most one of the $f_{d, m, i}$ is $t+1$, and hence at most one of the $p_{d, m, i}$ is $t+1$. So, $A_{\mu}$ has cyclic unipotent summand; hence by Proposition 5.7, $A_{\mu}$ is a maximal abelian subgroup. As $C_{\mathrm{GL}_{n}(q)}\left(g_{\mu}\right)$ is abelian and $A_{\mu} \subseteq C_{\mathrm{GL}_{n}(q)}\left(g_{\mu}\right)$, we get $A_{\mu}=C_{\mathrm{GL}_{n}(q)}\left(g_{\mu}\right)$.

Assume that $q \leq n$. We need to prove that $\mathcal{N}_{n}(q) \subset \mathcal{A}_{n}(q)$. Let $\mu_{0} \in \Phi_{n}$ be the map defined by

$$
\mu_{0}(d, m)= \begin{cases}0 & \text { if } d \geq 2 \text { or } m \geq 2 \\ n & \text { if } d=1 \text { and } m=1\end{cases}
$$

By Definition 5.1, $A_{\mu_{0}}$ is the group of diagonal matrices, that is, the split torus of size $(q-1)^{n}$. Since there are only $q-1$ distinct eigenvalues available, any $g \in A_{\mu_{0}}$ has an eigenvalue with multiplicity $\geq 2$. Therefore, $g$ is not a cyclic matrix, and $A_{\mu_{0}}$ contains no cyclic matrices. Thus $A_{\mu_{0}} \in \mathcal{A}_{n}(q) \backslash \mathcal{N}_{n}(q)$.

This allows us to complete the proof of Theorem 1.4.

Corollary $5.10 \omega\left(\mathrm{GL}_{n}(q)\right) \leq\left|\mathcal{A}_{n}(q)\right|$ with equality if and only if $q>n$.

Proof The inequality $\omega\left(\mathrm{GL}_{n}(q)\right) \leq\left|\mathcal{A}_{n}(q)\right|$ follows from Lemma 3.1 and Proposition 5.1. If $q>n$, then by Theorem 5.9 we have $\mathcal{A}_{n}(q)=\mathcal{N}_{n}(q)$. So, the inequality 
$\omega\left(\mathrm{GL}_{n}(q)\right) \geq\left|\mathcal{A}_{n}(q)\right|$ follows from Theorem 1.3. Now assume that $q \leq n$. Let $\mu_{0}$ be the function in $\Phi_{n}$ defined in (5.3), and $\mathcal{A}$ be the collection of subgroups $A$ of $\mathcal{A}_{n}(q)$ not conjugate to $A_{\mu_{0}}$. By Definition 5.1, $A_{\mu_{0}}$ is the group of diagonal matrices. Since there are only $q-1$ distinct eigenvalues available, every $g \in A_{\mu_{0}}$ has an eigenvalue with multiplicity $\geq 2$. Therefore, every $g$ is contained in some $A$, with $A \in \mathcal{A}$. Thus, from Proposition 5.1 we have $\operatorname{GL}_{n}(q)=\bigcup_{A \in \mathcal{A}} A$, and Lemma 3.1 yields $\omega\left(\mathrm{GL}_{n}(q)\right) \leq|\mathcal{A}|<\left|\mathcal{A}_{n}(q)\right|$.

Although Theorem 1.3 and Corollary 5.10 show that $N_{n}(q)$ and $\omega\left(\mathrm{GL}_{n}(q)\right)$ are bounded above by $\left|\mathcal{A}_{n}(q)\right|$, the value and order of magnitude of $\left|\mathcal{A}_{n}(q)\right|$ are not easy to establish from the definition of $\mathcal{A}_{n}(q)$. Therefore, in the next section, we determine (for $q>2$ ) a closed simple formula for the generating function $F(t)$ of $\left\{\left|\mathcal{A}_{n}(q)\right| /\left|\mathrm{GL}_{n}(q)\right|\right\}_{n \geq 1}$ (see Theorem 6.6). Also, in Sect. 7, by proving that $F(t)$ is analytic on a certain disk in the complex plane, we determine the asymptotic behaviour of $\left|\mathcal{A}_{n}(q)\right|$ as $n \rightarrow \infty$.

\section{A generating function}

We start by defining two generating functions.

Definition 6.1 Let $F(t)=\sum_{n=1}^{\infty} a_{n} t^{n}$ be the generating function for the proportion $a_{n}=\left|\mathcal{A}_{n}(q)\right| /\left|\mathrm{GL}_{n}(q)\right|$, and $\bar{F}(t)=\sum_{n=1}^{\infty} b_{n} t^{n}$ be the generating function for

$$
b_{n}=\sum_{\mu \in \Phi_{n}}\left|N_{\mathrm{GL}_{n}(q)}\left(A_{\mu}\right) \cap \operatorname{Stab}(V, \mu)\right|^{-1}
$$

(see Definitions 5.1 and 5.6).

Remark 6.2 By Lemma 5.3, we get

$$
a_{n}=\sum_{\mu \in \Phi_{n}}\left|N_{\mathrm{GL}_{n}(q)}\left(A_{\mu}\right)\right|^{-1},
$$

and so, by Definition 6.1, $a_{n} \leq b_{n}$ for every $n$. Furthermore, by Proposition 5.7, $F(t)=\bar{F}(t)$ for $q \geq 3$. In particular, $F(t)$ and $\bar{F}(t)$ differ only when $q=2$, in which case each coefficient $b_{n}$ of $\bar{F}(t)$ is an upper bound for the coefficient $a_{n}$ of $F(t)$. Since the generating function $\bar{F}(t)$ turns out to be easier to study, in the sequel we consider only $\bar{F}(t)$. This does not give rise to any restriction in the case of $q \geq 3$ and still provides an upper bound for $\left|\mathcal{A}_{n}(q)\right|$ when $q=2$.

We note that Corollary 5.10 yields $a_{n} \geq \omega\left(\mathrm{GL}_{n}(q)\right) /\left|\mathrm{GL}_{n}(q)\right|$ and, for $q>n$, $a_{n}=\omega\left(\mathrm{GL}_{n}(q)\right) /\left|\mathrm{GL}_{n}(q)\right|$. So, by studying $\bar{F}(t)$, we shall determine a good description for $\omega\left(\mathrm{GL}_{n}(q)\right)$. Namely, in Theorem 6.6 we prove a closed simple formula for the function $\bar{F}(t)$, and in Theorem 7.4 we give an exact formula for the limit $\lim _{n \rightarrow \infty} q^{n} b_{n}$.

Define the following two functions: 


$$
\begin{aligned}
& F_{1}(t)=\prod_{d \geq 1} \exp \left(\frac{t^{d}}{d\left(1-q^{-d}\right) q^{d}}\right), \\
& F_{2}(t)=\prod_{m \geq 2} \prod_{d \geq 1} \exp \left(\frac{t^{d m}}{d\left(1-q^{-d}\right)^{2} q^{2 d m-d}}\right) .
\end{aligned}
$$

Lemma 6.3 $\bar{F}(t)=F_{1}(t) F_{2}(t)$.

Proof From the Taylor series for the exponential function $\exp (t)$ and from Definition 6.2 we have

$$
\begin{aligned}
& F_{1}(t)=\prod_{d \geq 1}\left(\sum_{r=0}^{\infty} \frac{t^{d r}}{\left(d\left(1-q^{-d}\right) q^{d}\right)^{r} r !}\right), \\
& F_{2}(t)=\prod_{m \geq 2}\left(\prod_{d \geq 1}\left(\sum_{r=0}^{\infty} \frac{t^{d m r}}{\left(d\left(1-q^{-d}\right)^{2} q^{2 d m-d}\right)^{r} r !}\right)\right) .
\end{aligned}
$$

By expanding the infinite products in (6.3) for $F_{1}(t) F_{2}(t)$, in order to obtain a summand of degree $n$, we have to choose (for all $d, m \geq 1$ ) a term of degree $d m r_{d, m}$ from the series

$$
\sum_{r=0}^{\infty} \frac{t^{d r}}{\left(d\left(1-q^{-d}\right) q^{d}\right)^{r} r !} \quad(\text { for } m=1) \quad \text { or } \quad \sum_{r=0}^{\infty} \frac{t^{d m r}}{\left(d\left(1-q^{-d}\right)^{2} q^{2 d m-d}\right)^{r} r !}
$$

(for $m>1$ ),

in such a way that $n=\sum_{d, m} d m r_{d, m}$. This yields that each summand of degree $n$ obtained by expanding $F_{1}(t) F_{2}(t)$ is uniquely determined by an element $\mu \in \Phi_{n}$ (by setting $\left.\mu(d, m)=r_{d, m}\right)$. Hence, the coefficient of degree $n$ in $F_{1}(t) F_{2}(t)$ is

$$
\begin{aligned}
& \sum_{\mu \in \Phi_{n}}\left(\prod_{d \geq 1} \frac{1}{\left(d\left(1-q^{-d}\right) q^{d}\right)^{\mu(d, 1)} \mu(d, 1) !}\right. \\
& \left.\quad \times \prod_{d \geq 1, m \geq 2} \frac{1}{\left(d\left(1-q^{-d}\right)^{2} q^{2 d m-d}\right)^{\mu(d, m)} \mu(d, m) !}\right) .
\end{aligned}
$$

Applying Proposition 5.7 and (6.1), we see that the coefficient of $t^{n}$ in $\bar{F}(t)$ equals the coefficient of $t^{n}$ in $F_{1}(t) F_{2}(t)$. Thus $\bar{F}(t)=F_{1}(t) F_{2}(t)$.

In the rest of this section we use the theory of symmetric functions to obtain a closed simple formula for the generating functions $F_{1}(t)$ and $F_{2}(t)$. We start by recalling some well-known results and definitions from [11]. Let $X=\left\{x_{i}\right\}_{i \geq 1}$ be an infinite set of variables, and $\Lambda$ be the graded ring of symmetric functions on $X$ (see [11, Sect. I.2]). A partition is a sequence $\lambda=\left(\lambda_{1}, \lambda_{2}, \ldots\right)$ of non-negative integers in decreasing order and containing only finitely many non-zero terms. We write 
$|\lambda|=\sum_{i} \lambda_{i}$ and $X^{\lambda}=x_{1}^{\lambda_{1}} x_{2}^{\lambda_{2}} \cdots$. Let $\lambda$ be a partition. The function

$$
m_{\lambda}(X)=\sum_{\alpha} X^{\alpha}
$$

summed over all distinct permutations $\alpha$ of $\lambda$ is a symmetric function in $\Lambda$, and $m_{\lambda}$ is called a monomial symmetric function. By definition, $m_{\lambda}$ is a homogeneous function of degree $|\lambda|$. For each $d \geq 0$, the $d$ th complete symmetric function $h_{d}(X)$ is the sum of all monomial symmetric functions of degree $d$, so that

$$
h_{d}(X)=\sum_{|\lambda|=d} m_{\lambda}(X)
$$

The generating function for the complete symmetric functions is $H_{X}(t)=$ $\sum_{d \geq 0} h_{d}(X) t^{d}$ (the label $X$ in $H_{X}$ is needed in order to record the set of variables $X)$. It is proven in $[11,(2.5)$, p. 14] that

$$
H_{X}(t)=\prod_{i \geq 1}\left(1-x_{i} t\right)^{-1} .
$$

For each $d \geq 1$, the $d$ th power sum symmetric function is $p_{d}(X)=\sum_{i} x_{i}^{d}$. The generating function for the power sum symmetric functions is defined as $P_{X}(t)=$ $\sum_{d \geq 1} p_{d}(X) t^{d-1}$. It is proven in [11, (2.10), p. 16] that

$$
P_{X}(t)=\frac{d}{d t} \log H_{X}(t)
$$

From (6.5) we see that $k+\sum_{d \geq 1} p_{d}(X) t^{d} / d=\log H_{X}(t)$ for some integer $k$. Since $H_{X}(0)=h_{0}(X)=1$, we get that $\log H_{X}(0)=0$, and so $k=0$. Thence $H_{X}(t)=$ $\exp \left(\sum_{d} p_{d}(X) t^{d} / d\right)$. This shows that

$$
H_{X}(t)=\prod_{d \geq 1} \exp \left(\frac{p_{d}(X) t^{d}}{d}\right) .
$$

Write

$$
\varphi_{0}(x)=1 \quad \text { and } \quad \varphi_{d}(x)=(1-x) \cdots\left(1-x^{d}\right)
$$

for $d \geq 1$.

In the following lemma we establish two simple formulae for $F_{1}(t)$.

\section{Lemma 6.4}

(i) $F_{1}(t)=\prod_{i \geq 0}\left(1-q^{-(i+1)} t\right)^{-1}$.

(ii) $F_{1}(t)=\sum_{d=0}^{\infty} \frac{t^{d}}{q^{d} \varphi_{d}\left(q^{-1}\right)}$.

Proof Consider the set of variables $Q=\left\{q^{-(i-1)}\right\}_{i \geq 1}$, that is $Q$ is obtained by specialising $x_{i}=q^{-(i-1)}$ (for $i \geq 1$ ). From [11, Example 4, p. 19], we have that 
the $d$ th power sum symmetric function $p_{d}(Q)$ on the set of variables $Q$ satisfies $p_{d}(Q)=\left(1-q^{-d}\right)^{-1}$. So, (6.6) yields

$$
H_{Q}\left(q^{-1} t\right)=\prod_{d \geq 1} \exp \left(\frac{p_{d}(Q) t^{d}}{d q^{d}}\right)=\prod_{d \geq 1} \exp \left(\frac{t^{d}}{d\left(1-q^{-d}\right) q^{d}}\right)=F_{1}(t) .
$$

Equation (6.4) gives $H_{Q}\left(q^{-1} t\right)=\prod_{i \geq 1}\left(1-q^{-(i-1)}\left(q^{-1} t\right)\right)^{-1}=\prod_{i \geq 0}(1-$ $\left.q^{-(i+1)} t\right)^{-1}$, and $(i)$ follows.

From [11, Example 4, p. 19], we have that the $d$ th complete symmetric function $h_{d}(Q)$ on the set of variables $Q$ satisfies $h_{d}(Q)=1 / \varphi_{d}\left(q^{-1}\right)$. Thus, we obtain $H_{Q}(t)=\sum_{d=0}^{\infty} t^{d} / \varphi_{d}\left(q^{-1}\right)$ and $H_{Q}\left(q^{-1} t\right)=\sum_{d=0}^{\infty} t^{d} / q^{d} \varphi_{d}\left(q^{-1}\right)$, which, as we showed above, is $F_{1}(t)$, and (ii) is proved.

The argument for obtaining a simple formula for $F_{2}(t)$ is very similar to Lemma 6.4, but a little trickier. We start with a definition. For each $m \geq 2$, define

$$
F_{2}^{(m)}(t)=\prod_{d \geq 1} \exp \left(\frac{t^{d m}}{d\left(1-q^{-d}\right)^{2} q^{2 d m-d}}\right) .
$$

\section{Lemma 6.5}

(i) $F_{2}^{(m)}(t)=\prod_{i, j \geq 0}\left(1-q^{-(i+j+2 m-1)} t^{m}\right)^{-1}$.

(ii) $F_{2}(t)=\prod_{m \geq 2} \prod_{i, j \geq 0}\left(1-q^{-(i+j+2 m-1)} t^{m}\right)^{-1}$.

Proof Consider two infinite sets of variables $X=\left\{x_{i}\right\}_{i \geq 1}$ and $Y=\left\{y_{i}\right\}_{i \geq 1}$. From $X$ and $Y$ consider the infinite set of variables $Z=\left\{x_{i} y_{j}\right\}_{i, j \geq 1}$. By the definition of the $d$ th power sum symmetric function, we have

$$
\begin{aligned}
p_{d}(Z) & =\sum_{i, j \geq 1}\left(x_{i} y_{j}\right)^{d}=\sum_{i \geq 1} x_{i}^{d}\left(\sum_{j \geq 1} y_{j}^{d}\right) \\
& =\left(\sum_{i \geq 1} x_{i}^{d}\right)\left(\sum_{j \geq 1} y_{j}^{d}\right)=p_{d}(X) p_{d}(Y) .
\end{aligned}
$$

Consider the set of variables $Q=\left\{q^{-(i-1)}\right\}_{i \geq 1}$ obtained by specialising $x_{i}=q^{-(i-1)}$ (or $y_{i}=q^{-(i-1)}$ ) for $i \geq 1$. Also, consider the set of variables $Q^{\prime}=\left\{q^{-(i+j-2)}\right\}_{i, j \geq 1}$ obtained by specialising $x_{i} y_{j}=q^{-(i-1)} q^{-(j-1)}$. Under this assignment, we obtain from [11, Example 4, p. 19] and (6.9) that $p_{d}\left(Q^{\prime}\right)=p_{d}(Q)^{2}=\left(1-q^{-d}\right)^{-2}$.

Equation (6.6) yields

$$
\begin{aligned}
H_{Q^{\prime}}\left(q\left(q^{-2} t\right)^{m}\right) & =\prod_{d \geq 1} \exp \left(\frac{p_{d}\left(Q^{\prime}\right)\left(q\left(q^{-2} t\right)^{m}\right)^{d}}{d}\right)=\prod_{d \geq 1} \exp \left(\frac{p_{d}\left(Q^{\prime}\right) t^{d m}}{d q^{2 d m-d}}\right) \\
& =\prod_{d \geq 1} \exp \left(\frac{t^{d m}}{d\left(1-q^{-d}\right)^{2} q^{2 d m-d}}\right)=F_{2}^{(m)}(t)
\end{aligned}
$$


using (6.8). Finally, (6.4) gives

$$
\begin{aligned}
H_{Q^{\prime}}\left(q\left(q^{-2} t\right)^{m}\right) & =\prod_{i, j \geq 1}\left(1-q^{-(i+j-2)}\left(q\left(q^{-2} t\right)^{m}\right)\right)^{-1} \\
& =\prod_{i, j \geq 1}\left(1-q^{-(i+j+2 m-3)} t^{m}\right)^{-1}
\end{aligned}
$$

and (i) follows. Part (ii) follows from the definitions of $F_{2}$ and $F_{2}^{(m)}$ in (6.2) and (6.8).

The following theorem gives a formula for $\bar{F}(t)$.

\section{Theorem 6.6}

$$
\bar{F}(t)=\left(\prod_{i=0}^{\infty}\left(1-q^{-(i+1)} t\right)^{-1}\right)\left(\prod_{m \geq 2, i, j \geq 0}\left(1-q^{-(i+j+2 m-1)} t^{m}\right)^{-1}\right) .
$$

Proof As $\bar{F}(t)=F_{1}(t) F_{2}(t)$, the theorem follows from Lemmas 6.4 and 6.5.

By using the formula in Theorem 6.6 one can easily obtain the first few values of $b_{n}$, where $\bar{F}(t)=\sum_{n \geq 0} b_{n} t^{n}$. For instance, Table 1 in Sect. 1 was obtained by expanding the terms in $t$ of degree $\leq 6$ in the infinite products of $\bar{F}(t)$.

Using Lemma 6.4(ii), we show that $\left\{q^{n} b_{n}\right\}_{n \geq 0}$ is an increasing sequence.

Theorem 6.7 For each $n \geq 0, q^{n} b_{n}<q^{n+1} b_{n+1}$, where the $b_{n}$ are as in Definition 6.1. Moreover, if $q>2$, then the sequence $\left\{q^{n}\left|\mathcal{A}_{n}(q)\right| /\left|\mathrm{GL}_{n}(q)\right|\right\}_{n \geq 0}$ is increasing.

Proof Write $F_{2}(t)=\sum_{d \geq 0} c_{d} t^{d}$. It is clear from (6.3) that $c_{d} \geq 0$. By Lemma 6.4, we have $F_{1}(t)=\sum_{d \geq 0} \frac{t^{d}}{q^{d} \varphi_{d}\left(q^{-1}\right)}$ with $\varphi_{d}$ as in (6.7). Thus it follows from Lemma 6.3 that $b_{n}=\sum_{d=0}^{n} \frac{c_{n-d}}{q^{d} \varphi_{d}\left(q^{-1}\right)}$. Now $\varphi_{d+1}\left(q^{-1}\right)=\varphi_{d}\left(q^{-1}\right)\left(1-q^{-(d+1)}\right)<\varphi_{d}\left(q^{-1}\right)$ and $c_{d} \geq 0$, and hence

$$
\begin{aligned}
q^{n} b_{n} & =q^{n} \sum_{d=0}^{n} \frac{1}{q^{d} \varphi_{d}\left(q^{-1}\right)} c_{n-d}=\sum_{d=0}^{n} \frac{1}{\varphi_{d}\left(q^{-1}\right)}\left(q^{n-d} c_{n-d}\right) \\
& <\sum_{d=0}^{n} \frac{1}{\varphi_{d+1}\left(q^{-1}\right)}\left(q^{n-d} c_{n-d}\right)=q^{n+1} \sum_{d=0}^{n} \frac{1}{q^{d+1} \varphi_{d+1}\left(q^{-1}\right)} c_{n-d} \\
& =q^{n+1} \sum_{d=1}^{n+1} \frac{1}{q^{d} \varphi_{d}\left(q^{-1}\right)} c_{n+1-d} \leq q^{n+1} \sum_{d=0}^{n+1} \frac{1}{\varphi_{d}\left(q^{-1}\right)} c_{n+1-d}=q^{n+1} b_{n+1} .
\end{aligned}
$$

If $q>2$, then $b_{n}=\left|\mathcal{A}_{n}(q)\right| /\left|\mathrm{GL}_{n}(q)\right|$ by Remark 6.2 and the definition of $a_{n}$, and the last assertion follows. 


\section{Analytic properties of the generating function $\bar{F}(t)$}

Before studying analytically the functions $\bar{F}(t), F_{1}(t), F_{2}(t)$, we have to collect some numerical information that will be used later.

Lemma 7.1 Set $l(q)=\prod_{k=1}^{\infty}\left(1-q^{-k}\right)^{-k(k+1) / 2-1}$. We have:

(a) $l(q)>1+2 q^{-1}+7 q^{-2}+19 q^{-3}$.

(b) $l(q)<\left(1-q^{-1}-q^{-2}\right)^{-1} \exp \left(q^{-1} /\left(1-q^{-1}\right)^{3}\right) \exp \left(q^{-2}\left(1+q^{-1}\right) / 2\left(1-q^{-2}\right)^{4}\right)$.

(c) for $q>2, l(q)<1+2 q^{-1}+7 q^{-2}+114 q^{-3}$.

(d) if $q=2$, then $395.0005>l(2)>278.98$.

Proof Part (a) follows by expanding in powers of $q$ the first three terms $\left(1-q^{-1}\right)^{-2}$, $\left(1-q^{-2}\right)^{-4}$ and $\left(1-q^{-3}\right)^{-7}$ (for $\left.k=1,2,3\right)$ of the infinite product $l(q)$ and noticing that $\left(1-q^{-k}\right)^{-1}>1$, for $k \geq 1$.

Next, we consider an upper bound for $l(q)$. First, we recall that by the Binomial Theorem, $(1-x)^{-s}=\sum_{k=0}^{\infty}\left(\begin{array}{c}k+s-1 \\ s-1\end{array}\right) x^{k}$. In particular,

$$
\sum_{k=1}^{\infty}\left(\begin{array}{c}
k+1 \\
2
\end{array}\right) x^{k}=x \sum_{k=1}^{\infty}\left(\begin{array}{c}
k+1 \\
2
\end{array}\right) x^{k-1}=x \sum_{k^{\prime}=0}^{\infty}\left(\begin{array}{c}
k^{\prime}+2 \\
2
\end{array}\right) x^{k^{\prime}}=\frac{x}{(1-x)^{3}}
$$

Set $L(q)=\prod_{k=1}^{\infty}\left(1-q^{-k}\right)^{-k(k+1) / 2}$. We have

$$
\begin{aligned}
\log (L(q)) & =-\sum_{k=1}^{\infty} \frac{k(k+1)}{2} \log \left(1-q^{-k}\right)=\sum_{k=1}^{\infty} \frac{k(k+1)}{2}\left(\sum_{m=1}^{\infty} \frac{q^{-k m}}{m}\right) \\
& =\sum_{m=1}^{\infty} \frac{1}{m}\left(\sum_{k=1}^{\infty}\left(\begin{array}{c}
k+1 \\
2
\end{array}\right) q^{-k m}\right)=\sum_{m=1}^{\infty} \frac{q^{-m}}{m\left(1-q^{-m}\right)^{3}} \\
& <\frac{q^{-1}}{\left(1-q^{-1}\right)^{3}}+\frac{1}{2} \sum_{m=2}^{\infty} \frac{q^{-m}}{\left(1-q^{-m}\right)^{3}} \\
& <\frac{q^{-1}}{\left(1-q^{-1}\right)^{3}}+\frac{1}{2\left(1-q^{-2}\right)^{3}} \sum_{m=2}^{\infty} q^{-m} \\
& =\frac{q^{-1}}{\left(1-q^{-1}\right)^{3}}+\frac{q^{-2}}{2\left(1-q^{-2}\right)^{3}\left(1-q^{-1}\right)}
\end{aligned}
$$

where in $(\dagger)$ we used (7.1). From [14, Lemma 3.5] we have $\prod_{k=1}^{\infty}\left(1-q^{-k}\right)^{-1}<$ $\left(1-q^{-1}-q^{-2}\right)^{-1}$. Therefore, Part (b) follows.

Now, assume that $q>2$ and set $T=\exp \left(q^{-1} /\left(1-q^{-1}\right)^{3}\right)$. So,

$$
T=\sum_{r=0}^{\infty} \frac{\left(q^{-1} /\left(1-q^{-1}\right)^{3}\right)^{r}}{r !}<\sum_{r=0}^{3} \frac{\left(q^{-1} /\left(1-q^{-1}\right)^{3}\right)^{r}}{r !}+\sum_{r=4}^{\infty}\left(\frac{q^{-1} /\left(1-q^{-1}\right)^{3}}{2}\right)^{r}
$$




$$
=\sum_{r=0}^{3} \frac{\left(q^{-1} /\left(1-q^{-1}\right)^{3}\right)^{r}}{r !}+\frac{\left(\frac{q^{-1} /\left(1-q^{-1}\right)^{3}}{2}\right)^{4}}{1-\frac{q^{-1} /\left(1-q^{-1}\right)^{3}}{2}}<1+q^{-1}+\frac{7}{2} q^{-2}+41 q^{-3},
$$

where the first inequality uses $r ! \geq 2^{r}$ (for $r \geq 4$ ), and the last inequality is obtained by expanding in powers of $q$ and using the fact that $q>2$.

With similar computations we get $\exp \left(q^{-2}\left(1+q^{-1}\right) / 2\left(1-q^{-2}\right)^{4}\right)<1+q^{-2} / 2+$ $2 q^{-3}$ and $\left(1-q^{-1}-q^{-2}\right)^{-1}<1+q^{-1}+2 q^{-2}+7 q^{-3}$. Now, from Part (b) we have

$$
\begin{aligned}
l(q) & <\left(1+q^{-1}+2 q^{-2}+7 q^{-3}\right)\left(1+q^{-1}+\frac{7}{2} q^{-2}+41 q^{-3}\right)\left(1+\frac{1}{2} q^{-2}+2 q^{-3}\right) \\
& <1+2 q^{-1}+7 q^{-2}+114 q^{-3},
\end{aligned}
$$

and Part (c) follows.

The lower bound in Part (d) is obtained by computing $\prod_{k=1}^{30}\left(1-q^{-k}\right)^{-k(k+1) / 2-1}$ with $q=2$, and the upper bound is obtained by substituting $q=2$ in Part (b).

In the following two propositions, we study some analytic properties of $\bar{F}(t)$, $F_{1}(t), F_{2}(t)$.

Proposition 7.2 $F_{1}(t)$ is analytic on a disk of radius $q$. Also, $F_{1}(t)$ has a simple pole at $t=q$, and $\left(1-q^{-1} t\right) F_{1}(t)$ is analytic on a disk of radius $q^{2}$.

Proof From Definition 6.2 we obtain

$$
F_{1}(t)=\prod_{d=1}^{\infty} \exp \left(\frac{\left(q^{-1} t\right)^{d}}{d\left(1-q^{-d}\right)}\right)=\exp \left(\sum_{d=1}^{\infty} \frac{\left(q^{-1} t\right)^{d}}{d\left(1-q^{-d}\right)}\right) .
$$

Next, we determine where the series in (7.2) is absolutely convergent. We get

$$
\sum_{d=1}^{\infty} \frac{\left|q^{-1} t\right|^{d}}{d\left(1-q^{-d}\right)} \leq \sum_{d=1}^{\infty} \frac{\left|q^{-1} t\right|^{d}}{1-q^{-d}} \leq \frac{1}{1-q^{-1}} \sum_{d=1}^{\infty}\left|q^{-1} t\right|=\frac{\left|q^{-1} t\right|}{\left(1-q^{-1}\right)\left(1-\left|q^{-1} t\right|\right)}
$$

Since $1 /\left(1-q^{-1} t\right)$ is analytic on a disk of radius $q$ and has a simple pole in $t=q$, the equivalent result for $F_{1}(t)$ follows at once.

It remains to show that $\left(1-q^{-1} t\right) F_{1}(t)$ is analytic on a disk of radius $q^{2}$. Since $\varphi_{r}\left(q^{-1}\right)=\varphi_{r-1}\left(q^{-1}\right)\left(1-q^{-r}\right)$ (for $r \geq 1$ ), from Lemma 6.4(ii) we get

$$
\begin{aligned}
\left(1-q^{-1} t\right) F_{1}(t) & =\left(1-q^{-1} t\right) \sum_{r=0}^{\infty} \frac{t^{r}}{q^{r} \varphi_{r}\left(q^{-1}\right)} \\
& =1+\sum_{r=1}^{\infty}\left(\frac{t^{r}}{q^{r} \varphi_{r}\left(q^{-1}\right)}-\frac{t^{r}}{q^{r} \varphi_{r-1}\left(q^{-1}\right)}\right) \\
& =1+\sum_{r=1}^{\infty} \frac{q^{-r} t^{r}}{q^{r} \varphi_{r}\left(q^{-1}\right)}=\sum_{r=0}^{\infty} \frac{t^{r}}{q^{2 r} \varphi_{r}\left(q^{-1}\right)} .
\end{aligned}
$$


As

$$
\sum_{r=0}^{\infty} \frac{|t|^{r}}{q^{2 r} \varphi_{r}\left(q^{-1}\right)} \leq \prod_{i=1}^{\infty}\left(1-q^{-i}\right)^{-1} \sum_{r=0}^{\infty}\left|q^{-2} t\right|^{r}=\prod_{i=1}^{\infty}\left(1-q^{-i}\right)^{-1} \frac{1}{1-\left|q^{-2} t\right|},
$$

we get that $\left(1-q^{-1} t\right) F_{1}(t)$ is analytic on a disk of radius $q^{2}$.

Proposition 7.3 $F_{2}(t)$ is analytic on a disk of radius $q^{3 / 2}$.

Proof We argue as in Proposition 7.2. We have

$$
\begin{aligned}
F_{2}(t) & =\prod_{m=2}^{\infty} \prod_{d=1}^{\infty} \exp \left(\frac{t^{d m}}{d\left(1-q^{-d}\right)^{2} q^{2 d m-d}}\right)=\exp \left(\sum_{m=2}^{\infty} \sum_{d=1}^{\infty} \frac{t^{d m}}{d\left(1-q^{-d}\right)^{2} q^{2 d m-d}}\right) \\
& =\exp \left(\sum_{d=1}^{\infty} \frac{t^{2 d} q^{d}}{d\left(1-q^{-d}\right)^{2} q^{4 d}} \sum_{m=0}^{\infty} \frac{t^{d m}}{q^{2 d m}}\right) \\
& =\exp \left(\sum_{d=1}^{\infty} \frac{\left(q^{-2} t\right)^{2 d} q^{d}}{d\left(1-q^{-d}\right)^{2}\left(1-\left(q^{-2} t\right)^{d}\right)}\right) .
\end{aligned}
$$

Set $z=q^{-2} t$. We obtain

$$
\log \left(F_{2}(t)\right)=\sum_{d=1}^{\infty} \frac{z^{2 d} q^{d}}{d\left(1-q^{-d}\right)^{2}\left(1-z^{d}\right)} .
$$

We prove that for $|z|<q^{-1 / 2}$ (i.e. $|t|<q^{3 / 2}$ ), the series in (7.4) is absolutely convergent. Note that

$$
\left|\frac{1}{1-z^{d}}\right| \leq \frac{1}{1-q^{-d / 2}} \leq \frac{1}{1-q^{-1 / 2}} \quad \text { and } \quad \frac{1}{d\left(1-q^{-d}\right)^{2}} \leq \frac{1}{\left(1-q^{-1}\right)^{2}} .
$$

Thus the series in (7.4) is absolutely convergent if

$$
\sum_{d=1}^{\infty}\left|z^{2} q\right|^{d}=\frac{1}{1-\left|z^{2} q\right|}
$$

is absolutely convergent. As $|z|<q^{-1 / 2}$, we get $\left|z^{2} q\right|<1$, so $F_{2}(t)$ is analytic for $|t|<q^{3 / 2}$.

We finally determine the asymptotic behaviour of $\left\{b_{n}\right\}_{n \geq 1}$ (and so also for $\left\{a_{n}\right\}_{n \geq 1}$ when $q>2$ ).

Theorem 7.4 We have

$$
l(q)=\lim _{n \rightarrow \infty} q^{n} b_{n}=\prod_{k=1}^{\infty}\left(1-q^{-k}\right)^{-k(k+1) / 2-1}
$$

and also $\left|q^{n} b_{n}-l(q)\right|=o\left(r^{-n / 2}\right)$ for every $0<r<q$. 
Proof From Propositions 7.2 and 7.3 we get that $\bar{F}(t)$ is an analytic function on a disk of radius $q$, the point $t=q$ is a simple pole for $\bar{F}(t)$, and $f(t)=\left(1-q^{-1} t\right) \bar{F}(t)$ is an analytic function on a disk of radius $q^{3 / 2}$. In particular, by [10, Lemma 1.3.3], we get that $\lim _{n \rightarrow \infty} q^{n} b_{n}=f(q)$ and $\left|b_{n}-f(q) / q^{n}\right|=o\left(r^{-3 n / 2}\right)$ for every $0<r<q$. In particular, it remains to compute $f(q)$.

From Lemma 6.5(ii) we get $F_{2}(t)=\prod_{m \geq 2, i, j \geq 0}\left(1-q^{-(i+j+2 m-1)} t^{m}\right)^{-1}$. In particular, $F_{2}(q)=\prod_{m \geq 2, i, j \geq 0}\left(1-q^{-(i+j+m-1)}\right)^{-1}$. Now, given $k \geq 1$, there exist $k(k+1) / 2$ choices of $(i, j, m)$ such that $k=i+j+m-1$, with $m \geq 2$ and $i, j \geq 0$. Therefore, $F_{2}(q)=\prod_{k=1}^{\infty}\left(1-q^{-k}\right)^{-k(k+1) / 2}$.

From Lemma 6.4 we get $\left(1-q^{-1} t\right) F_{1}(t)=\prod_{i=1}^{\infty}\left(1-q^{-(i+1)} t\right)^{-1}$. So, $f(q)=$ $\prod_{i=1}^{\infty}\left(1-q^{-i}\right)^{-1} \prod_{k=1}^{\infty}\left(1-q^{-k}\right)^{-k(k+1) / 2}$, and the theorem follows.

Summing up, Theorem 1.6 follows from Theorems 6.7 and 7.4. Therefore, all the results mentioned in the introduction are now proved.

Remark 7.5 Since $\left|\mathrm{GL}_{n}(q)\right|=q^{n^{2}} \varphi_{n}\left(q^{-1}\right)$, Theorem 7.4 yields that $b_{n}\left|\mathrm{GL}_{n}(q)\right|$ is a polynomial $p(q)$ in $q$ of degree $q^{n^{2}-n}$. So, write $p(q)=\sum_{i=0}^{n^{2}-n} \alpha_{i} q^{n^{2}-n-i}$ and pick $r$ such that $0<r<q$.

As $\left|q^{n} b_{n}-l(q)\right|=o\left(r^{-n / 2}\right)$, we obtain that $\left|p(q)-q^{n^{2}-n} \varphi_{n}\left(q^{-1}\right) l(q)\right|=$ $o\left(r^{n^{2}-n-n / 2}\right)=o\left(r^{n^{2}-3 n / 2}\right)$. Furthermore, since $\varphi_{n}\left(q^{-1}\right) l(q)=\prod_{k=n+1}^{\infty}(1-$ $\left.q^{-k}\right)^{-1} \prod_{k=1}^{\infty}\left(1-q^{-k}\right)^{-k(k+1) / 2}$, we see that

$$
\left|\sum_{i=0}^{n^{2}-n} \alpha_{i} q^{n^{2}-n-i}-q^{n^{2}-n} \prod_{k=1}^{\infty}\left(1-q^{-k}\right)^{-k(k+1) / 2}\right|=o\left(r^{n^{2}-3 n / 2}\right) .
$$

It follows that the first $\lfloor n / 2\rfloor$ coefficients $\alpha_{0}, \ldots, \alpha_{\lfloor n / 2\rfloor-1}$ are obtained from the series expansion of $\prod_{k=1}^{\infty}\left(1-q^{-k}\right)^{-k(k+1) / 2}$. Such a sequence is described and studied in [17].

Remark 7.6 It was conjectured in [2] that, for $q>n$,

$$
\omega\left(\mathrm{GL}_{n}(q)\right) \geq q^{n^{2}-n}+\frac{\left|\mathrm{GL}_{n}(q)\right|}{q(q-1)^{n}}+\frac{\left|\mathrm{GL}_{n}(q)\right|}{q^{\left(n^{2}-n\right) / 2}(q-1)^{2}} .
$$

The second summand on the right-hand side of this inequality is asymptotic to $q^{n^{2}-n-1}+(n-1) q^{n^{2}-n-2}+O\left(q^{n^{2}-n-3}\right)$. Therefore, Remark 7.5 and Table 1 yield that this conjecture is incorrect for $n \geq 6$.

\section{Concluding comments}

We conclude by noticing that we can exploit the theory presented in this paper further in order to obtain a refinement of Corollary 5.10 in the case that $q \leq n$. We give an example in the case $n=q$ and $q>2$. 
From the proof of Theorem 5.9 we have that if $n=q$, then all $A_{\mu}$ (for $\mu \in$ $\left.\Phi_{n}\right)$ are centralisers of cyclic matrices except if $\sum_{m} \mu(1, m)>q-1$. Since $q=$ $\sum_{d, m} \mu(d, m) d m$, this implies $\sum_{m} \mu(1, m)=q$, and so $\mu=\mu_{0}$, where

$$
\mu_{0}(d, m)= \begin{cases}0 & \text { if } d \geq 2 \text { or } m \geq 2 \\ q & \text { if } d=1 \text { and } m=1\end{cases}
$$

By Definition 5.1, $A_{\mu_{0}}$ is the group of diagonal matrices, that is, the split torus of size $(q-1)^{q}$. By Proposition 5.7 we have $\left|N_{\mathrm{GL}_{q}(q)}\left(A_{\mu_{0}}\right)\right|=(q-1)^{q} q$ !.

Since there are only $q-1$ distinct eigenvalues available, any $g \in \mathrm{GL}_{q}(q)$ giving a decomposition of $V_{g}$ as $\bigoplus_{i=1}^{q} V_{i}$ into one-dimensional spaces has an eigenvalue with multiplicity $\geq 2$. Therefore, we see that $g$ is centralised by some cyclic matrix $g_{\mu} \in A_{\mu}$ for $\mu \neq \mu_{0}$. In particular, following the proof of Corollary 5.10, we have that

$$
\omega\left(\mathrm{GL}_{q}(q)\right)=\left|\mathcal{A}_{q}\right|-\frac{\left|\mathrm{GL}_{q}(q)\right|}{(q-1)^{q} q !} .
$$

It is not clear to the authors of this paper whether there is a general theory for small $q$ and large $n$ with a tractable formula for $\omega\left(\mathrm{GL}_{n}(q)\right)$.

\section{References}

1. Abdollahi, A., Akbari, A., Maimani, H.R.: Non-commuting graph of a group. J. Algebra 298, 468-492 (2006)

2. Azad, A., Praeger, C.E.: Maximal sets of pairwise noncommuting elements of finite three-dimensional general linear groups. Bull. Aust. Math. Soc. 80, 91-104 (2009)

3. Bertram, E.A.: Some applications of graph theory to finite groups. Discrete Math. 44, 31-43 (1983)

4. Brown, R.: Minimal covers of $S_{n}$ by abelian subgroups and maximal subsets of pairwise noncommuting elements. J. Comb. Theory, Ser. A 49(2), 294-307 (1988)

5. Carter, R.W.: Finite Groups of Lie Type, Conjugacy Classes and Complex Characters. Wiley, Chichester (1993)

6. Chin, A.M.Y.: On non-commuting sets in an extra special p-group. J. Group Theory 8, 189-194 (2005)

7. Curtis, C.W., Reiner, I.: Representation Theory of Finite Groups and Associative Algebras. Pure and Applied Mathematics, vol. XI. Wiley, New York (1962)

8. Dixon, J.D.: Maximal abelian subgroups of the symmetric groups. Can. J. Math. 23, 426-438 (1971)

9. Fulman, J.: Cycle indices for the finite classical groups. J. Group Theory 2, 251-289 (1999)

10. Fulman, J., Neuman, P.N., Praeger, C.E.: A Generating Function Approach to the Enumeration of Matrices in Classical Groups over Finite Fields. Mem. AMS, vol. 176. AMS, Providence (2005)

11. Macdonald, I.G.: Symmetric Functions and Hall Polynomials. Clarendon Press, Oxford (1979)

12. Faber, V., Laver, R., Mckenzie, R.: Covering of groups by abelian subgroups. Can. J. Math. 30, $933-$ 945 (1978)

13. Neumann, B.H.: A problem of Paul Erdôs on groups. J. Aust. Math. Soc. A 21, 467-472 (1976)

14. Neumann, P.M., Praeger, C.E.: Cyclic matrices over finite fields. J. Lond. Math. Soc. 52, 263-284 (1995)

15. Neumann, P.M., Praeger, C.E.: Cyclic matrices and the MEATAXE. In: Ohio State Univ. Math. Res. Inst. Publ., vol. 8, pp. 291-300. de Gruyter, Berlin (2001)

16. Pyber, L.: The number of pairwise non-commuting elements and the index of the centre in a finite group. J. Lond. Math. Soc. 35, 287-295 (1987)

17. Sloane, N.J.A. (ed.): The On-Line Encyclopedia of Integer Sequences. Published electronically at www.research.att.com/ njas/sequences/SequenceA000294 (2008) 
18. Tomkinson, H.J.: Groups covered by finitely many cosets or subgroups. Commun. Algebra 15, $845-$ 859 (1987)

19. Vdovin, E.P.: The number of subgroups with trivial unipotent radicals in finite groups of Lie type. J. Group Theory 7, 99-112 (2004)

20. Wall, G.E.: Counting cyclic and separable matrices over a finite field. Bull. Aust. Math. Soc. 60, 253-284 (1999) 